\title{
Marke und Markenkommunikation
}

Die Kapitel 2, 3 und 4 legen die theoretischen Grundlagen dieser Arbeit dar, die den Einfluss von Brand Content als eine Form der Markenkommunikation auf das Markenimage untersucht. ${ }^{1}$ Dazu werden in diesem Kapitel die Begriffe Marke, Markenidentität, Markenimage, Markenpositionierung und Markenkommunikation beleuchtet. Obwohl der Begriff der Marke im allgemeinen Sprachgebrauch tief verankert ist und Menschen auf Nachfrage mühelos beispielhafte Marken wie UBS, Nestlé, Nike, Adidas oder Apple nennen können, birgt die wissenschaftliche Auseinandersetzung mit dem Begriff einige Herausforderungen (Baumgarth, 2014, S. 1). Ursprünglich beschrieb der Begriff «Marke» ein Symbol, mit dem ein Unternehmen seine Produkte oder Waren kennzeichnete und damit deren Herkunft und Qualität auszeichnete (Hellmann, 2003). Aus mindestens drei Gründen greift eine solche Begriffsbestimmung heute zu kurz:

Erstens ist die Marke aus Sicht des Unternehmens heute nicht mehr ein Kennzeichen, das dem Produkt am Ende des Produktionsprozesses hinzugefügt wird, sondern ein umfassendes Leitmotiv unternehmerischen Handelns. Die Marke gibt als handlungsleitendes Konzept vor, welche Produkte ein Unternehmen sinnvollerweise in welcher Art und Qualität herstellt und wie diese an welche Abnehmer

\footnotetext{
${ }^{1}$ Über ein Markenimage verfügen (früher oder später) prinzipiell alle Menschen, die mit einer Marke in Kontakt kommen. Die Gesamtheit dieser Menschen bilden die Anspruchsgruppen bzw. sie sind Stakeholder des Unternehmens oder der Marke. Das heisst, sie nehmen in irgendeiner Form Einfluss auf die Unternehmenstätigkeit oder werden durch diese beeinflusst (Freeman, 1984, S. 46). Kundinnen, Kunden und Konsumierende kaufen die Produkte oder Dienstleistungen der Marke. Sie bilden die marktorientierte(n) Zielgruppe(n) der Marke (Tropp, 2019, S. 267). Schliesslich wird oft der Begriff des Users oder der Userin verwendet. Damit sind Kundinnen oder Kunden, aber auch sonstige Mitglieder der Anspruchsgruppen gemeint, die über digitale Kanäle mit einer Marke interagieren.
} 
vermarktet werden. Ansätze des Brand Managements bzw. der Markenführung widmen sich dieser strategischen Perspektive (Esch, 2012, S. 83).

Zweitens wird der Begriff Marke nicht mehr nur in Zusammenhang mit Produkten oder Waren, sondern in Zusammenhang mit diversen Bezugsobjekten wie Unternehmen, Organisationen, Dienstleistungen, Personen oder Ländern gebraucht (Buhmann \& Ingenhoff, 2015; Ingenhoff \& Fuhrer, 2010). Diese Arbeit fokussiert aus einer wirtschaftlichen Perspektive auf Marken, die sich auf Unternehmen (Unternehmensmarke wie z. B. Nestlé) oder spezifische Produkte eines Unternehmens (Produktmarke wie z. B. Nescafé) beziehen.

Drittens trägt heute Kommunikation einen Grossteil dazu bei, was eine Marke ausmacht. Die Unternehmen setzen Markenkommunikation (z. B. Werbung) ein, um die Marke (bzw. das Bezugsobjekt der Marke) bekannt zu machen und diese in ein positives Licht zu rücken. Konsumierende berichten ihrerseits über ihre Erlebnisse mit der Marke und veröffentlichen Fotos und Videos, wie sie diese im Alltag nutzen (usergenerierte Inhalte). Die Marke hat sich dadurch vom Kennzeichen gelöst und ist zu einem umfassenderen Konstrukt geworden, dessen Ausgestaltung, Wahrnehmung und Interpretation zu einem wesentlichen Teil durch Kommunikation geprägt wird (Hellmann, 2003, S. 89-92; Zerfass, 2010, S. 389). «Marken entstehen durch Kommunikation [Hervorhebung im Original]» (S. 4), schreiben Esch und Honal (2018) diesbezüglich. Mit diesem dritten Punkt beschäftigt sich diese Arbeit.

Bevor in Abschnitt 2.3.1 eine detaillierte Bestimmung des Begriffs «Marke» und der damit verbundenen Elemente erfolgt, sollen in Abschnitt 2.1 verschiedene Ansätze zur Marke präsentiert werden (Heding et al., 2009). Diese geben als «Denkschulen» (S. 22) einen Überblick, was unter einer Marke verstanden werden kann und wie sich das Konstrukt der Marke im Zeitlauf verändert hat (Heding et al., 2009). Zudem stecken sie den Rahmen ab, wie und wozu das

Konstrukt der Marke Anwendung findet. Weil die Übersicht allerdings nur am Rande auf soziale Medien eingeht, wird die digitale Kommunikationslandschaft in einem weiteren Kapitel betrachtet.

\subsection{Ansätze und Denkschulen in Bezug auf Marke}

Heding et al. (2009, S. 20) identifizieren für den Zeitraum von 1985 bis 2006 zwei Paradigmen, drei Zeitperioden und sieben Ansätze oder Denkschulen in Bezug darauf, wie das Konzept der Marke verstanden und umschrieben wird. 
Im positivistischen Paradigma besitzt der Markeninhaber die vollständige Kontrolle über die Marke und deren Kommunikation. Er bestimmt über den Wert ${ }^{2}$ der Marke, also darüber, was die Marke ausmacht. Die Konsumentinnen und Konsumenten werden als passive Rezipierende verstanden. Dem konstruktivistischen oder interpretativen Paradigma liegt hingegen die Annahme aktiver Konsumierender zugrunde. Die Marke bzw. deren Wesen oder Wert wird im Zusammenspiel zwischen Konsumentinnen und Konsumenten und Markeninhaber erzeugt. Der Paradigmenwechsel vollzieht sich schleichend in den 1990er-Jahren (Heding et al., 2009, S. 21).

Weiter unterscheiden die Autoren zwischen drei verschiedenen Zeitperioden. In der ersten Zeitperiode (1985 bis 1992) stehen das Unternehmen und die von ihm gesteuerten Marketing-massnahmen im Zentrum. Die passiven Rezipierenden nehmen die Marketingbotschaften in der vom Markeninhaber intendierten Form auf. Wenn in der Kommunikation alle Elemente richtig justiert sind, kann Brand Equity kreiert und die Konsumierenden können in ihrem Verhalten beeinflusst werden (Heding et al., 2009, S. 22). Im Jahr 1993 beginnt mit dem Artikel von Keller «Conceptualizing, Measuring, and Managing Customer-Based Brand Equity» (1993) eine zweite Zeitperiode, die bis 1999 dauert. In diesen sechs Jahren steht der Rezipient im Mittelpunkt des Brand Managements (S. 23). Brand Equity wird nicht mehr durch den Markeninhaber kreiert, sondern entsteht in den Köpfen der Konsumentinnen und Konsumenten. Eine dritte Zeitperiode, die mit dem Jahr 2000 anbricht, wurde durch neue Muster des Markenkonsums initiiert, die wiederum auf technologische und kulturelle Veränderungen zurückzuführen sind. Schlagworte wie Brand Communitys ${ }^{3}$, Anti-Branding-Bewegungen oder Brand Icons, Marken mit hoher kultureller Bedeutung (Holt, 2004, S. 11), spielen hier eine zentrale Rolle (Heding et al., 2009, S. 24-25).

\footnotetext{
${ }^{2}$ Heding et al. (2009) benutzen in ihren Erklärungen den Begriff «Brand Equity». Damit wird einerseits der finanzielle, buchhalterische Wert der Marke gemeint und andererseits - im Sinne Kellers «Customer-Based Brand Equity» - die positive Wahrnehmung der Marke unter den Ziel- und Anspruchsgruppen (siehe Heding et al., 2009, S. 11).

${ }^{3}$ Muniz und O'Guinn (2001, S. 412) definieren Brand Community als «a specialized, nongeographically bound community, based on a structured set of social relationships among admirers of a brand». Communitys haben insbesondere im digitalen Raum an Bedeutung und Verbreitung gewonnen. Wenn Userinnen und User auf Webseiten, Plattformen oder in sozialen Netzwerken zusammenkommen, um sich über eine spezifische Marke auszutauschen, wird von einer Brand Community gesprochen. Es kann zwischen unternehmensinitiierten Communitys (z. B. die Fans einer Marke auf Facebook) und userinitiierten Communitys (z. B. private Facebook-Gruppe, die sich um eine Marke dreht) unterschieden werden (von Loewenfeld, 2006).
} 
Schliesslich unterscheiden die Autoren in einem weiteren Detaillierungsgrad zwischen sieben verschiedenen Ansätzen des Brand Managements (Heding et al., 2009). Die Ansätze schaffen eine Ordnungs- und Interpretationsbasis für die Arbeit und sollen deshalb hier kurz vorgestellt werden. Die Angaben stammen - falls nicht anders angegeben - von Heding et al. (2009):

\section{Der Economic Approach}

Der ökonomische Ansatz (Heding et al., 2009, S. 29-46) geht davon aus, dass die Markenkontrolle vollständig dem Markeninhaber obliegt. Mit einer optimalen Steuerung des Marketings und der Kommunikation kann der Konsumierende zu einem gewünschten Verhalten gebracht werden. In der Tradition der neoklassischen Wirtschaftstheorie wird der Konsumierende als rational handelnder, sich perfekt informierender Mensch verstanden, der die Marketingbotschaften rezipiert, evaluiert und anschliessend so handelt, dass sich sein Nutzen maximiert (Heding et al., 2009, S. 32). Mit dem ökonomischen Ansatz beginnt die Entkoppelung des Brand Managements vom Marketingmanagement, und die Etablierung eines eigenständigen Theorie- und Forschungsfeldes setzt ein (Heding et al., 2009, S. 23).

\section{Der Identity Approach}

Der identitätsbasierte Ansatz (Heding et al., 2009, S. 47-82) wurde stark durch die Praxis vorangetrieben. Die Anfänge liegen in den Bemühungen, eine Marke durch ein konsequentes, gleichbleibendes Erscheinungsbild (Corporate Identity) zu stärken. Dabei spielen sowohl die visuelle Identität (Visual Identity) als auch das Verhalten der Mitarbeitenden (Behavioural Identity) eine zentrale Rolle. Eine Marke und die Mitarbeitenden müssen in allen Aktivitäten und über alle Markenberührungspunkte (Marken-Touchpoints) ein kohärentes, auf einer starken inneren Identität beruhendes Erlebnis bieten, damit ein positives und klares Vorstellungsbild (Markenimage) unter den Zielgruppen entstehen kann.

\section{Der Consumer-Based Approach}

Der Consumer-Based Approach (Heding et al., 2009, S. 83-115) ist bis heute der am meisten verwendete und der einflussreichste Ansatz, um das Konzept der Marke zu klären. Er nahm seinen Lauf mit dem Artikel «Conceptualizing, Measuring, and Managing Customer-Based Brand Equity» von Keller im Jahr 1993. Brand Equity entsteht demnach in den Köpfen der Zielgruppen. Damit hielten die kognitive Psychologie und Prozesse der Informationsverarbeitung Einzug in das Markenmanagement. Das Ziel der Markenführung liegt darin, bei den Konsumierenden Brand Equity durch «starke, einzigartige und positive» (Heding 
et al., 2009, S. 85) Markenassoziationen aufzubauen. Nach Heding et al. (2009) rückt zwar der Konsument in den Mittelpunkt; dieser wird aber nach wie vor als «maschineller» (S. 154) Rezipierender verstanden: Mit einer entsprechenden Botschaft kann der Markeninhaber beim Konsumenten eine intendierte Reaktion auslösen.

\section{Der Personality Approach}

Dem Personality Approach (Heding et al., 2009, S. 116-150) liegt die Annahme zugrunde, dass Konsumierende Marken menschliche Züge bzw. Eigenschaften zuschreiben. Die Konsumierenden wählen diejenige Marke aus, mit der sie ihre eigene Identität am besten ausdrücken können. In der Markenführung werden spezifische Charakteristika in den Vordergrund gerückt, um die Marke über Persönlichkeitsmerkmale zu differenzieren. Die Beziehung zwischen Marke und Konsument ist zunehmend zweiseitig geprägt (Heding et al., 2009, S. 120). Ana$\log \mathrm{zu}$ den fünf menschlichen Persönlichkeitsmerkmalen der Menschen leitete J. L. Aaker (1997) fünf Dimensionen der Markenpersönlichkeit ab: Aufrichtigkeit, Erregung/Spannung, Kompetenz, Kultiviertheit und Robustheit (J. L. Aaker, 2005).

\section{Der Relational Approach}

Der beziehungsorientierte Ansatz (Heding et al., 2009, S. 151-180) besagt, dass Menschen mit Marken Beziehungen eingehen, so wie sie es mit anderen Menschen tun. Die Ausgestaltung der Beziehung ist von Individuum zu Individuum verschieden, folgt aber immer einem Zweck. Das Erlebnis des Konsumenten mit der Marke und nicht die funktionale Zweckerfüllung des Produkts steht im Vordergrund. Für Heding et al. (2009, S. 173) ist der Relational Approach der erste Ansatz, bei welchem der Sinngehalt (meaning) einer Marke und nicht die Information (Marke als Sender) im Vordergrund steht. Dieser Sinngehalt individueller Beziehungen wird kontinuierlich zwischen Konsument und Marke ko-konstruiert (S. 171, 175). Heding et al. (2009) schreiben: «It seems that brand management in a way <lets go〉 of the brand via this approach. The brand is suddenly <out there> in a chaotic and ever-changing context» (S. 175). Mit diesem Ansatz rücken erstmals der Kunde und sein Wesen gesamtheitlich in den Fokus (S. 173) - im Gegensatz etwa zum Customer-Based Approach, in welchem der Konsument als passiver Rezipient verstanden wird (S. 154).

\section{Der Community Approach}

Die bisherigen Ansätze fokussierten auf die Beziehung zwischen der Marke und einem einzelnen Konsumenten. Mit dem Community Approach (Heding et al., 
2009, S. 181-206) weitet sich die Perspektive aus und Beziehungen zwischen den Konsumenten einer Marke gewinnen an Beachtung. Aus dyadischen (Marke Kunde) werden triadische Markenbeziehungen (Marke - Kunde - Kunde; Heun, 2014b; Muñiz \& O'Guinn, 2001). Der Sinngehalt einer Marke wird nicht mehr individuell, sondern in Gruppen (z. B. Brand Communitys) ko-kreiert. Der Markeninhaber ist bei diesem Prozess nicht mehr zwingend dabei. Das kann so weit führen, dass er die Hoheit über die Marke an eine Community verliert. Auf der anderen Seite gelten Community-Mitglieder als loyal gegenüber der Marke und als potenzielle Quelle für Weiterempfehlungen, innovative Ideen oder Verbesserungsvorschläge (Heding et al., 2009, S. 182).

\section{Der Cultural Approach}

Beim Cultural Approach (Heding et al., 2009, S. 207-242) wird die Marke in den gesellschaftlich-kulturellen Kontext eingebettet und als «kulturelles Artefakt» (Heding et al., 2009, S. 212) verstanden. Die kulturelle Bedeutung der Produkte (für den Konsumierenden) und nicht das Produkt an sich steht im Fokus (S. 223). Im kulturellen Ansatz spielen zwei gegensätzliche Perspektiven eine Rolle (Heding et al., 2009, S. 208): Auf der einen Seite geht es darum, wie kulturelle Einflüsse den Aufstieg von Marken zu globalen, «ikonischen» Supermarken begünstigen und wie Marken wiederum selbst zum Kulturgut beitragen. Auf der anderen Seite geht es um die negativen Auswirkungen von Marken - und Unternehmen generell - auf die Kultur, Gesellschaft und Umwelt. Als grundlegender Autor gilt Holt (2004).

Diese Ansätze zeigen auf, wie sich das Verständnis und der Geltungsbereich der Marke seit den 1980er-Jahren verändert haben. Allerdings werden die Implikationen, die sich durch die Digitalisierung der Kommunikationslandschaft für das Markenmanagement ergeben, nicht explizit behandelt. Obwohl viele der dargestellten Mechanismen (zweiseitige Beziehungen, Communitys etc.) gerade auch in der digitalen Welt ihre Gültigkeit haben, soll im nächsten Schritt die Marke im Kontext der digitalen Kommunikationslandschaft eingeordnet werden. Denn gerade die Digitalisierung der Kommunikationslandschaft hat das Wesen der Marke und die Markenkommunikation wesentlich geprägt. Erst unter Berücksichtigung dieser zusätzlichen Informationen kann in Abschnitt 2.3.1 definiert werden, was als Marke im Rahmen dieser Arbeit verstanden werden soll. 


\subsection{Marke im Kontext der digitalen Kommunikation}

Marken entstehen zu einem Grossteil in einem mediatisierten Kontext. Dieser Umstand, der mit der Reklame seinen Ursprung nahm (Hellmann, 2003), findet in einer digitalisierten Welt seinen Höhepunkt (Pleil \& Zerfass, 2014, S. 732). Persönliche Erfahrungen mit einem Hersteller oder Verkäufer werden zunehmend durch Webseiten, Social-Media-Posts oder Sprachassistenten abgelöst. Zum Wesen der digitalen Kommunikationslandschaft haben insbesondere das Internet mit den sozialen Medien und sozialen Netzwerken beigetragen (Kietzmann, Hermkens, McCarthy \& Silvestre, 2011).

\subsubsection{Internet und soziale Medien}

In Anlehnung an Beck (2014) kann das Internet als ein «technisches Medium erster Ordnung» (S. 4) verstanden werden, welches unter Nutzung einer physikalischen Infrastruktur (Server, Leitungen etc.) die «technische Plattform» (S. 4) für internetspezifische «Medien zweiter Ordnung» (S. 4) wie das World Wide Web, Webseiten, Chats, soziale Medien (Social Media) oder soziale Netzwerke stellt. Im Zusammenhang mit internetspezifischen Medien zweiter Ordnung fallen oft die Begriffe Web 1.0 und Web 2.0. Das World Wide Web entstand auf der Basis des Web 1.0 (A. M. Kaplan \& Haenlein, 2010). Dieses zeichnete sich dadurch aus, dass User auf existierende Inhalte zugreifen konnten (Schenk \& Jers, 2013, S. 70). Das Web 2.0 kann als Weiterentwicklung des Web 1.0 angesehen werden. Diese Weiterentwicklung ist weniger technischer als vielmehr sozialer Art. Erhöhte Interaktivität und gesteigerte Benutzerfreundlichkeit sind die beiden elementaren Vorzüge des Web 2.0 gegenüber dem Web 1.0 (Portmann \& Meier, 2010, S. 277). Mit dem Web 2.0 wurden die Userinnen und User befähigt, selbst Inhalte verfügbar zu machen, zu teilen und zu verbreiten (Schenk \& Jers, 2013, S. 70). Damit wurde die Basis für die sozialen Medien gelegt. Entsprechend werden in der weit verbreiteten Definition nach Kaplan und Haenlein (2010) soziale Medien auf der Grundlage von Web 2.0 wie folgt umschrieben:

Social Media is a group of Internet-based applications that build on the ideological and technological foundations of Web 2.0, and that allow the creation and exchange of User Generated Content. (A. M. Kaplan \& Haenlein, 2010, S. 61)

Mit dem Begriff des User Generated Content (usergenerierte Inhalte) sind jene Inhalte, Reviews, Meinungen oder Diskussionen gemeint, die von Userinnen und 
Usern ausserhalb professioneller Tätigkeit erstellt und online über die unterschiedlichsten sozialen Medien zugänglich gemacht werden (Daugherty, Eastin \& Bright, 2008, S. 16; Owusu, Mutshinda, Antai, Dadzie \& Winston, 2016, S. 23). Der Begriff soziale Medien hat damit eine sehr grosse Reichweite. Mast (2019, S. 374) nennt Blogs, Twitter, Facebook oder YouTube als Beispiele sozialer Medien. Wang, Tchernev und Solloway (2012) subsumieren soziale Netzwerke (z. B. Facebook), Kommunikationstools (z. B. E-Mail) und Seiten, die zum Teilen und Kommentieren von Informationen genutzt werden (z. B. YouTube), unter den sozialen Medien. Soziale Netzwerke können entsprechend als Teilmenge der sozialen Medien verstanden werden. Sie zeichnen sich primär dadurch aus, dass die User ein eigenes Profil erstellen und sich mit anderen Usern verlinken können (Beck, 2014, S. 21; Boyd \& Ellison, 2008, S. 211). Die Abgrenzung der Begriffe ist allerdings nicht immer trennscharf. In der Definition von sozialen Medien nach Correa et al. (2010, S. 248) spielt beispielsweise die Vernetzung der Userinnen und User ebenfalls eine zentrale Rolle.

Im Rahmen dieser Arbeit stehen insbesondere soziale Netzwerke im Fokus. Damit sind Applikationen auf Basis des Web 2.0 gemeint, bei denen sich User und Userinnen, aber auch Unternehmen ein Profil anlegen, sich untereinander vernetzen und unternehmensgenerierte und usergenerierte Inhalte austauschen. Die Begriffe soziale Medien und Social Media werden synonym verwendet. Oft wird im weiteren Verlauf bedeutungsgleich auch der Begriff der digitalen Kanäle verwendet. Blogs, E-Mail oder Wikis, die teilweise ebenfalls den sozialen Medien zugeordnet werden, spielen im Rahmen dieser Arbeit keine primäre Rolle.

\subsubsection{Zweiseitige Kommunikation in sozialen Medien}

Das Verständnis von Kommunikation hat sich in den letzten 120 Jahren kontinuierlich, aber insbesondere durch das Aufkommen bzw. die zunehmende Relevanz der sozialen Medien seit der Jahrtausendwende stark verändert. Am Anfang des 20. Jahrhunderts dominierte ein einseitiges Kommunikationsverständnis mit allmächtigen Sendern und den für Kommunikationsbotschaften empfänglichen Empfängern (Jäckel, 2011, S. 77). Medien oder Marken steuerten den Kommunikationsprozess und übermittelten ihre Botschaften an die passiven Kunden, die als Empfänger in der vom Kommunikator intendierten Weise reagierten (Jäckel, 2011, S. 78; A. Mitchell, 2012). Ein solches Verständnis ist heute kaum mehr relevant. Die Kommunikation hat sich von einem primär einseitigen hin zu einem vielseitigen, dialogorientierten Prozess gewandelt (Schögel \& Mrkwicka, 2011, 
S. 6). Das Bild vom Individuum als passivem Empfänger änderte hin zum selbstbestimmten Akteur, der eingebettet in sein soziales Umfeld handelt (Burkart, 2019, S. 170; Potthoff, 2016).

Obwohl sich dieser Wechsel bereits ab dem frühen 20. Jahrhundert kontinuierlich vollzogen hat (Jäckel, 2011, S. 83), kumuliert diese Entwicklung in einer digitalen Kommunikationslandschaft. Jeder Mensch kann heute über digitale Medien ein potenzielles Millionenpublikum finden und auf Augenhöhe mit Marken und Unternehmen interagieren. Einzelpersonen können Marken mittels auf Facebook, Weibo oder Twitter vermittelter, kritischer Botschaften in Bedrängnis bringen (Grégoire, Salle \& Tripp, 2015). Ebenso bedeutend ist die Summe aggregierter Meinungen, wie sie auf Hotelbuchungsplattformen oder Online-Shops über Erfolg oder Misserfolg von Produkten und Dienstleistungen bestimmen. Die Kommunikation unter Usern ist zu einem wesentlichen Faktor für Marken geworden (Heun, 2014b). Aktivitäten, Geschichten und Inhalte von Marken werden geteilt und verbreiten sich rasch und mit hoher Reichweite. Beziehungen und Austauschprozesse innerhalb von Anspruchs- oder Kundengruppen, ohne aktive Anteilnahme des Markeninhabers, werden immer zahlreicher. Wenn sich Kunden im Kaufentscheidungsprozess über Marken und Produkte informieren, dann greifen sie in ihrer Entscheidungsfindung massgeblich auf Empfehlungen anderer Kunden zurück (PwC, 2018; K. Z. Zhang, Zhao, Cheung \& Lee, 2014, S. 78). Markeninhaber mussten die Deutungshoheit über die Marke zu einem gewissen Grad abgeben (Gensler, Völckner, Liu-Thompkins \& Wiertz, 2013, S. 243). Markenkommunikation muss deshalb heute vor dem Hintergrund dreiseitig (triadisch) strukturierter Beziehungen (User-User-Marke) anstelle dyadisch strukturierter Beziehungen (User-Marke) verstanden werden (Heun, 2014b, S. 2).

Damit nehmen User heute beispielsweise beim «Brand Storytelling» eine aktive Rolle ein (Singh \& Sonnenburg, 2012): Es ist nicht mehr allein am Unternehmen als Markeninhaber, über Themen, Inhalt, Produktion und Distribution der Markenkommunikation zu entscheiden. «Brand Stories» (S. 189) geben einen unternehmensinitiierten thematischen Rahmen vor, um welchen herum sich Konversationen zwischen Usern und dem Unternehmen als Markeninhaber bilden. Usergenerierte Inhalte können dabei in Inhalt und Form mehr oder weniger von der durch den Markeninhaber intendierten Markenstory abweichen. Singh und Sonnenburg (2012) sehen in dieser Interaktion zwischen Markeninhaber und Anspruchsgruppen Parallelen zu einem Improvisationstheater, bei dem sich die Geschichte ebenfalls durch die Interaktion von Darstellern und Publikum ergibt. Gensler et al. (2013) kommen vor dem Hintergrund usergenerierter Inhalte zum selben Fazit: «The construction of brands can thus be interpreted as a collective, co-creational process [eigene Hervorhebung] involving several brand authors who 
all contribute their stories» (S. 244). Das Konzept der Ko-Kreation entstammt ursprünglich der Innovationsforschung (Hatch \& Schultz, 2010) und behandelt die gemeinsame Wertschöpfung zwischen Konsumenten und Produzenten (Prahalad \& Ramaswamy, 2004). Ko-Kreation kann Produkte und Dienstleistungen, aber auch Prozesse, die Distribution oder symbolische Aspekte wie die Marketingbotschaften betreffen (Payne, Storbacka, Frow \& Knox, 2009, S. 379; Pongsakornrungsilp \& Schroeder, 2011, S. 305; Prahalad \& Ramaswamy, 2004, S. 5). Mit der zunehmenden Bedeutung usergenerierter Inhalte wurde der Begriff der Ko-Kreation auch auf die Marke angewendet. Iglesias, Ind und Alfaro (2013) entwickeln die «organic brand view» als ein «brand value co-creation model». Dabei verstehen sie Marken als «organic entities» (S. 671), die im Zusammenspiel mit verschiedenen Stakeholdern entstehen. Die Markeninhaber können zwar die Richtung der Markengestaltung vorgeben, aber nicht allein darüber bestimmen, wofür diese steht (Iglesias et al., 2013).

Diese Ausführungen zu sozialen Medien und der zweiseitigen Kommunikation sollen zeigen, dass digitale Kanäle wesentlich dazu beigetragen haben, dass sich das «Konstrukt» der Marke verändert hat. Marken werden heute sowohl durch den Markeninhaber als auch durch User geprägt.

\subsection{Grundlagen und Definition der Marke}

Im folgenden Kapitel wird der Begriff der Marke beschrieben. Danach wird auf wesentliche Elemente der Marke eingegangen: Markenidentität, Markenimage, Markenpositionierung und Markenkommunikation.

Abbildung 2.1 zeigt bildhaft, wie die verschiedenen Markenelemente in Verbindung stehen. Unternehmens- oder Produktmarken, wie sie im Rahmen dieser Arbeit betrachtet werden, haben ihren Ursprung in einem Unternehmen, werden aber zunehmend durch User beeinflusst. Eine Marke bewegt sich deshalb sowohl in einem unternehmensinternen als auch in einem öffentlichen Raum. Der unternehmensinterne Raum obliegt primär der Kontrolle des Unternehmens. Allerdings kann auch dort usergenerierte Markenkommunikation stattfinden, wenn sich Mitarbeitende in einem informellen Rahmen über die eigenen Produkte austauschen. Insbesondere durch die Digitalisierung hat das Unternehmen einen Kontrollverlust im öffentlichen Raum über die eigene «Marke» erfahren. Usergenerierte Inhalte, die ausserhalb des Einflussbereichs des Unternehmens liegen, sind dort zunehmend omnipräsent, konkurrieren die unternehmensgenerierten Markenkommunikation und tragen wesentlich dazu bei, wie eine Marke wahrgenommen wird (Markenimage). 


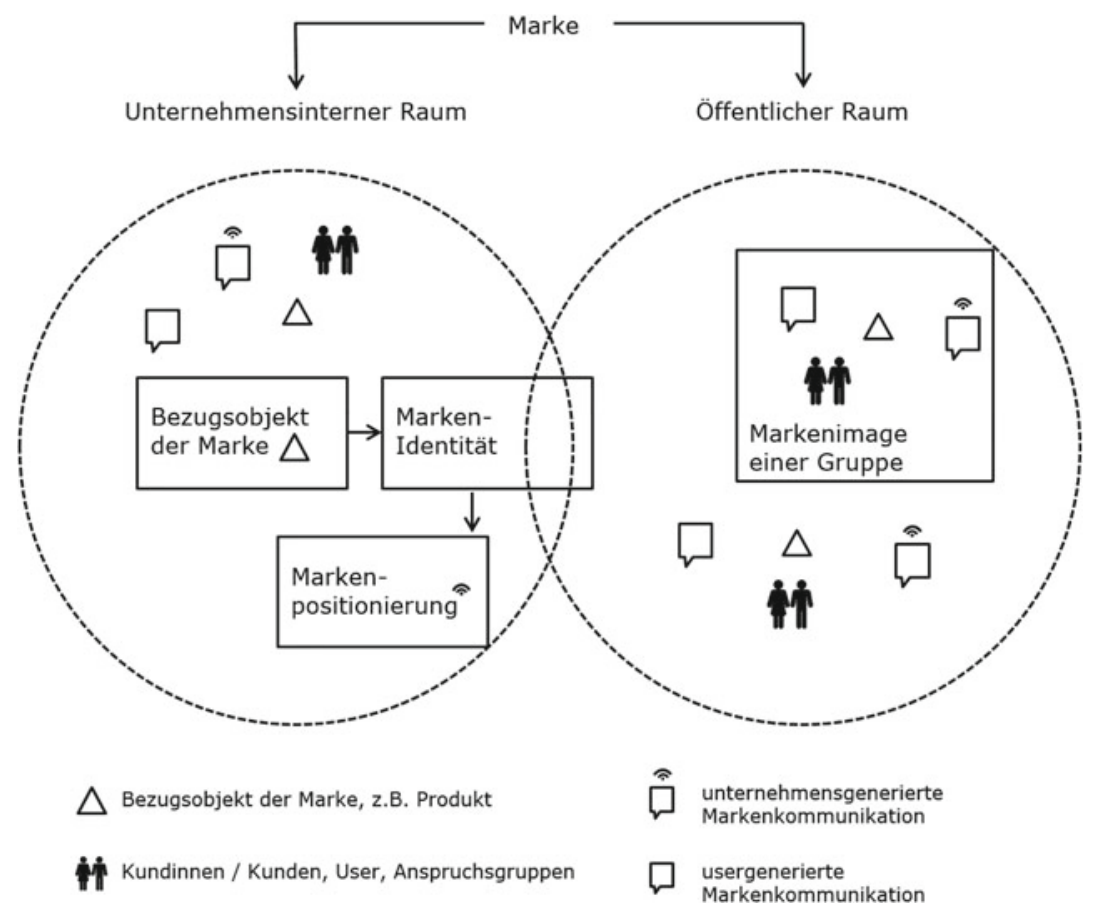

Abbildung 2.1 Marke und Markenelemente. (Eigene Darstellung)

\subsubsection{Marke}

Unter Zuhilfenahme der präsentierten Ansätze in Bezug auf Marken (Heding et al., 2009) und unter Berücksichtigung der Implikationen der digitalen Kommunikationslandschaft soll in diesem Kapitel ein dieser Arbeit zugrundeliegendes Markenverständnis entwickelt werden. Dazu werden primär Überlegungen aus dem Identity Approach, dem Consumer-Based Approach, dem Relational Approach und dem Community Approach berücksichtigt. Im Anschluss werden die Konstrukte Markenidentität, Markenimage, Markenpositionierung und Markenkommunikation geklärt.

Einleitend zu diesem Kapitel wurde geschrieben, dass der Begriff Marke ursprünglich als Zeichen zur Kennzeichnung von Produkten und Waren verstanden wurde. Es wurde dann aufgezeigt, dass diese Interpretation nicht mehr 
zeitgemäss ist und Marken heute zu einem Grossteil das Resultat von Kommunikation sind. Wie genau das Konstrukt Marke zu definieren ist, darüber gehen die Meinungen und Auffassungen in der Literatur dennoch auseinander (Kapferer, 2012, S. 9). Nach Esch (2012) sind Marken «Vorstellungsbilder in den Köpfen der Anspruchsgruppen, die eine Identifikations- und Differenzierungsfunktion übernehmen und das Wahlverhalten prägen» (S. 22). Die Marke wird hier vom Unternehmen losgekoppelt und existiert nur noch als Vorstellungsbild aufseiten der Anspruchsgruppen. Diese Definition folgt eng dem Consumer-Based Approach nach Keller (1993).

Eine gegenseitige Perspektive nimmt die Markenberatungsfirma Interbrand ein. In der «Brand Strategy Toolbox» (Interbrand, 2012) wird die Marke definiert als «a living business asset, brought to life across all touchpoints which, if properly managed, creates identification, differentiation and value». Die Marke ist demnach ein Vermögenswert, der sich auf Unternehmensseite findet und eng mit dem Identity Approach verwandt. Die Marke wird durch das Unternehmen gesteuert und an den diversen Touchpoints bewusst zum Ausdruck gebracht. Im Gegensatz zu Esch (2012), der die Marke prinzipiell bei Kunden verortet, wird die Marke hier vor allem beim Unternehmen verortet. Ein ähnliche Perspektive nehmen Niederhäuser und Rosenberger (2017) ein. Für sie ist die Marke ein «kommunikative[s] Versprechen [Hervorhebung im Original]» (S. 37) bzw. ein Vehikel zur «Selbstbeschreibung» (S. 38) des Unternehmens.

Auf Basis der bisherigen Ausführungen scheint es jedoch nicht sinnvoll, die Marke einseitig auf der Unternehmens- oder der Userseite zu verorten. Wenn die Marke zu einem Grossteil das Resultat von Kommunikation ist, dann werden zwangsläufig zwei Seiten benötigt, um eine Marke entstehen zu lassen. Kommunikation nämlich ist «ein zweiseitiger Prozess [eigene Hervorhebung], in dem die Beteiligten durch Mitteilungs- und Verstehenshandlungen jeweils neue Wirklichkeiten schaffen» (Zerfass \& Piwinger, 2014, S. 1). Entsprechend wurde in Abschnitt 2.2.2 dargelegt, dass im Sinne von Ko-Kreation heute sowohl die Unternehmen als Markeninhaber als auch die diversen User zur Entstehung der Marke beitragen. Diese Reziprozität kommt insbesondere im Relational Approach oder im Community Approach zum Tragen. Beim Relational Approach beispielsweise werden der Sinngehalt der Marke, also wofür eine Marke steht, wesentlich durch den Konsum und die Nutzung der Marke mitbestimmt (Heding et al., 2009, S. 155). Allerdings spricht auch Fournier (1998), die Hauptvertreterin des Ansatzes, von der Marke «als Partner» in der Beziehung Kunde - Marke (S. 344), was impliziert, dass die Marke auch ohne Zutun des Kunden bereits existieren muss. Der wesentliche Beitrag des Community-Ansatzes liegt darin, dass Gruppen ein gemeinsames Verständnis und gemeinsame Rituale in Bezug auf 
ein «Branded Product» (Muñiz \& O'Guinn, 2001, S. 412) bilden. Dabei kann das Unternehmen als Markeninhaber beim markenbezogenen Austausch unter den Mitgliedern mehr oder weniger involviert sein kann (von Loewenfeld, 2006, S. 127). Gerade bei userinitiierten Brand Communitys spielt das Unternehmen an sich keine Rolle, abgesehen davon, dass es das Produkt produziert, um welches sich die Community dreht.

Ein Mittelweg, bei dem sowohl dem Unternehmen als auch dem User eine aktive Rolle zukommt, findet sich etwa beim Ansatz der identitätsbasierten Markenführung (Burmann, Halaszovich, Schade \& Hemmann, 2015; Burmann et al., 2018). Dort wird Marke definiert als «ein Bündel aus funktionalen und nichtfunktionalen Nutzen, deren Ausgestaltung sich aus Sicht der Zielgruppen der Marke nachhaltig gegenüber konkurrierenden Angeboten differenziert» (Burmann et al., 2015, S. 28). Diese Verbindung einer «internen Ursachenperspektive» mit einer externen «Wirkungsperspektive» (Burmann et al., 2015, S. 28) sehen die Autoren als Vorteil ihrer Definition. Während die auf Produkt- oder Markeneigenschaften basierenden «intendierten Nutzen» aus Sicht des Markeninhabers die Ursachenperspektive abbilden, ergibt sich die Wirkungsperspektive durch die Interpretation oder Einschätzung der Zielgruppen der Nutzen der Marke. Der internen Perspektive widmet sich im identitätsbasierten Ansatz die Markenidentität, der externen Perspektive das Markenimage. Deigendesch (2013) schreibt in ähnlicher Weise: «Marken entstehen erst im rekursiven Verhältnis zwischen Identität und Markenimage» (S. 770).

Die Definition der identitätsorientierten Markenführung bietet eine gute Ausgangslage, wirft aber noch einige Unklarheiten auf:

- Einerseits ist fraglich, ob sich eine Marke einzig über die Ausgestaltung von Nutzen bildet. Personen, die einer Marke kritisch gegenüberstehen, würden zwar deren Nutzen, wohl aber nicht die Marke selbst leugnen.

- Damit einhergehend ist auch der Begriff der Zielgruppe zu eng gefasst. Mit Zielgruppe werden primär die potenziellen und bestehenden Kundinnen und Kunden umschrieben, also die Abnehmer der Produkte und Dienstleistungen von Marken und Unternehmen (Kloss, 2012, S. 194). Die Anwendung des Markenkonstrukts beschränkt sich jedoch nicht auf Märkte, sondern hat Relevanz in einer breiten Öffentlichkeit.

- Schliesslich werden die Personen in der Zielgruppe als primär passive Akteure verstanden, die sich höchstens ein Markenimage auf Basis einer Interpretation vorgegebener Nutzen bilden. Gerade diese Passivität ist aber in einer digitalisierten Welt kritisch zu hinterfragen. 
Es braucht den bisherigen Überlegungen zufolge mindestens ein Produkt, eine Dienstleistung oder ein anderes Bezugsobjekt (Bruhn, 2014, S. 44), worauf sich die Marke beziehen kann. Die «Marke» dieses Bezugsobjekts entsteht im Rahmen eines zweiseitigen, kommunikativen Ausdifferenzierungsprozesses zwischen dem Unternehmen als Markeninhaber und den unterschiedlichen Anspruchsgruppen bzw. Usern. Dabei muss markenbezogene Kommunikation mitgeteilt, wahrgenommen und verarbeitet werden (Schmid \& Lyczek, 2008, S. 7; Zerfass \& Piwinger, 2014, S. 1). Demzufolge soll für den Rahmen dieser Arbeit Folgendes festgehalten werden: Eine Marke konstruiert sich als die Summe individueller Vermittlungs- und Wahrnehmungsleistungen gegenüber einem markierten Bezugsobjekt. Die Marke umfasst damit gemäss Abbildung 2.1 ein Bezugsobjekt und alle dazugehörenden markenbezogenen Tätigkeiten im unternehmensinternen und öffentlichen Raum. Individuelle Vermittlungsleistungen werden nach wie vor oft vom Markeninhaber, immer stärker aber auch von Usern erbracht, die in vielfältiger Weise über verschiedenste Bezugsobjekte kommunizieren und damit zu deren Marke beitragen.

Diese Definition soll helfen, den Begriff der Marke umfassend zu verstehen und markenbezogene Mechanismen in einem grösstmöglichen Kontext zu betrachten. Bei einer Einschränkung des Markenbegriffs auf eine Innen- oder Aussensicht wäre das nicht möglich. Der Einfachheit halber wird das Wort Marke teilweise weiterhin als Subjekt verwendet, z. B. wenn «die Marke auf sozialen Medien kommuniziert». Marke steht dann stellvertretend für das Unternehmen, das Eigentümer der Marke ist bzw. diese führt. Nach der Klärung des Markenbegriffs können in den nächsten Kapiteln einzelne Markenelemente diskutiert werden.

\subsubsection{Markenidentität}

Der Begriff der Identität beschreibt, was eine Organisation ist (Hatch \& Schultz, 1997, S. 357). Es kann zwischen einer organisatorischen und einer marketingorientierten Perspektive unterschieden werden. Die organisationale Identität beschreibt, wie Mitarbeitende eine Organisation «wahrnehmen», «einschätzen» und wie sie über die Organisation «denken» (Hatch \& Schultz, 1997, S. 357). Der Begriff der Corporate Identity in der Tradition der marketingorientierten Perspektive hingegen befasst sich primär damit, wie das Management ein ideales Bild dessen, was die Organisation sein soll, an die Anspruchsgruppen vermitteln kann (Hatch \& Schultz, 1997, S. 357). 
Übertragen auf den Markenkontext ist die Markenidentität eine Festlegung dessen, wofür eine Marke steht bzw. stehen soll. Die Markenidentität umschreibt die Herkunft, die Werte, den Zweck oder die Essenz der Marke (Esch, 2012, S. 81). Die Markenidentität definiert also «das Selbstbild der Marke» (Burmann et al., 2015, S. 29) aus Unternehmenssicht, wobei das Selbstbild diejenigen Merkmale umfasst, «die aus Sicht der internen Zielgruppen in nachhaltiger Weise den Charakter der Marke prägen» (Burmann et al., 2015, S. 29). Sie ist ein strategisches Orientierungs- oder Steuerungsinstrument, um die Marke im Markt zu etablieren. Madhavaram et al. (2005) beschreiben die Markenidentität als «the brand concept from the brand strategist's perspective» (S. 76). Für D. A. Aaker und Joachimsthaler (2000) ist die Markenidentität «a set of associations the brand strategist seeks to create or maintain» (S. 40). Der Ursprung der Identität eines Unternehmens oder einer Marke findet sich damit in der Unternehmenspolitik und der damit verbundenen, grundlegenden Aufgabe des Unternehmens (Niederhäuser \& Rosenberger, 2017, S. 8).

Im vorherigen Kapitel wurde dargelegt, dass eine Marke das Resultat eines ko-kreativen Austauschprozesses zwischen Unternehmen und Usern ist. Betrachtet man die Markenidentität als jene Bausteine, welche die Marke prägen und ausmachen, dann drängt sich die Frage auf, ob die Markenidentität nach wie vor im Unternehmen bzw. beim Markeninhaber verortet werden kann. Oder kokreiert sich die Markenidentität ebenfalls unter Einflussnahme externer User oder Anspruchsgruppen? Die Meinungen dazu fallen unterschiedlich aus. Auf der einen Seite finden sich Konzepte, die, wie oben erwähnt, Identität als «organisationale Selbstbeschreibung» (S. 434) verstehen (Eisenegger, 2015). Eisenegger nennt hier beispielhaft auch die Definition von Markwick und Fill (1997): «Corporate identity is the organization's presentation of itself to its various stakeholders and the means by which it distinguishes itself from all other organizations» (S. 397). In vergleichbarer Weise kann die Definition von Balmer und Greyser (2006) eingeordnet werden, wonach die Identität darüber Auskunft gibt, «what we indubitably are» (S. 735). Schliesslich versteht auch Ingenhoff (2018) unter Identität die Art und Weise, wie «interne Stakeholders die Organisation oder Einheit konstruieren oder wahrnehmen» (S. 4).

Auf der anderen Seite finden sich Aussagen, nach denen auch die Markenidentität das Resultat eines gemeinsamen Austauschprozesses ist. Kornum, Gyrd-Jones, Al Zagir und Brandis (2017) beschreiben mit Brand Identity beispielsweise ein sozial konstruiertes Phänomen, das aus der Interaktion zwischen Individuen, Stakeholdern und dem Unternehmen hervorgeht. Ihre Erkenntnisse gehen auf eine ethnografische Studie innerhalb einer Nike-orientierten Running Community zurück. Die Resultate zeigen, dass Nike und die Running Community 
ähnliche Prinzipien vertreten, z. B. durch eine Performance-Orientierung oder die fast ausschliessliche Nike-Kleidung in der Community. Durch sogenanntes orthogonales Verhalten (z. B. Rauchen, Alkohol) grenzen sich die Mitglieder aber auch von Nike-Werten ab. Für die Autoren liegt der Erfolg der Community und auch für Nike, die die Community mit Vergünstigungen unterstützt, darin, dass Nike solches orthogonales Verhalten toleriert.

$\mathrm{Zu}$ ähnlichen Ergebnissen kommen auch Black und Veloutsou (2017), die sich mit der «Yes Edinburgh North \& Leith»-Kampagne, einem Teil der nationalen Referendumskampagne «Yes Scotland» zur schottischen Unabhängigkeit, beschäftigen. Die Autoren zeigen, wie freiwillige Helfer der «Yes Edinburgh North \& Leith»-Kampagne zu einer Eigendynamik innerhalb der «Yes Scotland»Kampagne verhelfen, die sich beispielsweise durch ein angepasstes Logo, einen angepassten Claim oder eigene Symbole manifestiert. Auf diese angepasste Symbolik griff danach auch die übergeordnete Marke «Yes Scotland» zurück. Die Autoren schreiben:

Its use [the Fiona graphic] by different local groups changed the national brand by modifying the identity of its constituent parts, making the brand less centralized and more chaotic and reinforcing its grassroots nature. (Black \& Veloutsou, 2017, S. 424)

The findings suggest that when consumers interact with brands, they do not just co-create brand production; they also create the brand identity, contribute to brand reputation, and express their identity through their active support of the branded offer. (Black \& Veloutsou, 2017, S. 426)

Auch Heding et al. (2009, S. 248) kommen zum Fazit, dass dem identitätsorientierten Ansatz zu Beginn eine senderdominierte Perspektive zugrunde lag, inzwischen jedoch ko-kreative Prozesse, in denen die Identität zwischen Markeninhaber und Konsumierenden entsteht, an Bedeutung gewonnen hätten. Es mag für gewisse Marken gelten, dass einflussreiche Anspruchs- oder Kundengruppen tatsächlich wesentlich mitbestimmen, wofür eine Marke im Kern steht. Im Normalfall ist allerdings davon auszugehen, dass (nach wie vor) primär der Markeninhaber oder der «Brand Strategist» über die Ausrichtung der Markenidentität bestimmt. Aber selbst dann nehmen externe Anspruchsgruppen durchaus Einfluss darauf, wie eine Markenidentität durch den Markeninhaber ausgestaltet wird (Gioia, Schultz \& Corley, 2000).

Eine solcher Prozess wird nachvollziehbar durch den Ansatz der Negotiated Brand (Gregory, 2007). Zwar nimmt die Autorin nicht direkt Bezug auf Markenidentität, sondern beschreibt die Entwicklung von Marken insgesamt. Unter dem Wort «Marke» wird aber insbesondere die Ausgestaltung von Werten oder 
die strategische Vision der Marke diskutiert. Insofern lassen sich diese Ausführungen auf die Konstruktion einer Markenidentität übertragen. Beim Ansatz der «Negotiated Brand» wird davon ausgegangen, dass die Marke in Zusammenarbeit entsteht. Durch Dialog und Verhandlung zwischen dem Markeninhaber und externen Anspruchsgruppen entwickelt sich die Marke über den Lauf der Zeit weiter. Die Initiative zur Markengestaltung geht dabei vom Unternehmen (einer Organisation) aus, das in einem Prozess die für das Unternehmen bzw. die Marke bedeutenden Werte ausarbeitet. Über die Unternehmenskommunikation gelangen die Werte an die Stakeholder, welche die Werte beurteilen. Basierend auf dem informellen Feedback können Massnahmen zur Korrektur vorgenommen werden (Gregory, 2007, S. 63). Die Autorin betont, dass sich die durch diesen Austauschprozess entstehenden Marken zwar konstant weiterentwickeln, auf Basis eines grundlegenden Werte-Sets - «a set of values that will remain consistent (although evolving) over time» (S. 64) -, aber über die Zeit hinweg beständig und unverkennbar bleiben (Gregory, 2007). Von geringen Anpassungen bis hin zum langfristigen Austausch einzelner Kernwerte ist dabei alles möglich. So fasst die Autorin entsprechend zusammen, dass der Grad der Stakeholder-Involvierung von der Ausgestaltung der Organisation abhängt (Gregory, 2007). Während bei Konsumgütermarken die Stakeholder-Involvierung geringer ausfalle, sei diese bei Marken mit einem sozialen Zweck wohl ausgeprägter.

Zusammenfassend lässt sich festhalten, dass Marken eine Identität oder einen langfristigen, konsistenten Kern benötigen (Iglesias et al., 2013, S. 683). Die Identität beschreibt das Wesen der Marke, hält als Sollbild fest, wofür diese stehen soll, und gibt deren strategische Stossrichtung vor (Esch, 2012, S. 81). Sie determiniert die Markenpositionierung, die Markenkommunikation und das Markenimage. Sie wird primär durch den Markeninhaber, allerdings unter Berücksichtigung äusserer Einflüsse, festgelegt. Das verlangen nicht zuletzt auch die Kunden. Edelman (2010) schreibt beispielsweise: «Consumers still want a clear brand promise and offerings they value» (o. S.). In Abbildung 2.1 wird die Markenidentität deshalb primär im unternehmensinternen, aber teilweise auch im öffentlichen Raum verortet.

\subsubsection{Markenimage}

Während die Identität das Sollbild der Marke darstellt, umschreibt das Markenimage die Wahrnehmung einer Marke bzw. eines markierten Produkts durch 
die unterschiedlichen internen und externen Anspruchsgruppen. Die Wahrnehmung von «Dingen» ist eine soziale Konstruktion ${ }^{4}$ (Schmid \& Lyczek, 2008, S. 9). Was beispielsweise ein Produkt ist, liegt nicht nur am Produkt selbst, sondern insbesondere daran, was die Gesellschaft aus dem Produkt macht oder eben konstruiert. Dieser sozial-konstruktivistische Blick erklärt, warum sich die Wahrnehmung und Bedeutung von Produkten (z. B. Erdöl, Automobil, Atomstrom) über den Zeitverlauf oder über Kulturkreise hinweg ändern kann (Schmid \& Lyczek, 2008, S. 9). Dann ist die Wahrnehmung von Produkten aber auch individuell geprägt. Während Nike-Schuhe bei einer Person A der bequemen Fortbewegung dienen, drückt eine andere Person B durch das Tragen derselben Schuhe eine urbane, stilorientierte Lebensorientierung aus. Schmid und Lyczek schreiben, dass Konsumenten ein «inneres Bild» (Schmid \& Lyczek, 2008, S. 51) eines Produkts haben, und meinen damit das mit dem Produkt verbundene Wissen. Dieses innere Bild kann auch als Image bezeichnet werden (Schmid \& Lyczek, 2008, S. 52). Zwar verwenden Schmidt und Lyczek in ihren Beschreibungen das Wort «Produkt», die Ausführungen können aber auch auf Marken übertragen werden, insbesondere weil die Autoren selbst nicht explizit zwischen den Begriffen unterscheiden und übergreifendes, markenunabhängiges Wissen zur Produktkategorie als «generische Image[s]» (S. 53) bezeichnen.

Das Image einer Marke ergibt sich einerseits durch direkte Erfahrungen (insbesondere durch die Nutzung des Produkts) und bildet sich andererseits über Kommunikation (Esch, 2019, S. 909). Imageprägende Kommunikationsinhalte - das wurde in Abschnitt 2.2.2 dargelegt - stammen dabei sowohl vom Unternehmen selbst als auch von diversen weiteren Quellen wie Medien, Bekannten, Community-Mitgliedern, Internet-Usern etc. (Schmid \& Lyczek, 2008, S. 97). Dieser Umstand wurde entsprechend auch in Abbildung 2.1 berücksichtigt. Das Markenimage bezieht sich dort zudem nur auf eine Auswahl an Kundinnen und Kunden. Das soll verdeutlichen, dass ein Markenimage je nach Individuum anders ausfallen kann. Gerade starke Marken verfügen allerdings auch in der Summe - über alle Anspruchsgruppen, User und Kunden und Kundinnen hinweg - über ein konsistentes, in weiten Teilen gleichbleibendes Image (Esch, 2019, S. 911; Keller, 2013, S. 453). Ein starkes, konsistentes Markenimage ist aus Unternehmenssicht aus verschiedenen Gründen wichtig (Burmann et al., 2018, S. 48-50; Deigendesch, 2013; Ingenhoff, 2018; Park, Jaworski \& MacInnis, 1986).

\footnotetext{
${ }^{4} \mathrm{Zu}$ den Grundlagen des Sozialkonstruktivismus siehe Berger und Luckmann (1966).
} 
1. Es sorgt für eine hohe Wiedererkennung und vermittelt rasch eine Vielzahl an Informationen. Dadurch werden Komplexität und Unsicherheit im Kaufentscheidungsprozess reduziert und Vertrauen aufgebaut.

2. Es unterstützt Kunden dabei, der eigenen Persönlichkeit Ausdruck zu verleihen.

3. Es weckt positive Emotionen und gibt den Konsumierenden ein gutes Gefühl.

4. Es begünstigt die Differenzierung gegenüber der Konkurrenz.

5. Es wirkt sich positiv auf den Kauf der Produkte und auf die Kundenloyalität aus.

6. Es dient der Legitimität des Unternehmens im gesellschaftlich-sozialen Kontext.

7. Es sorgt für Motivation und Identifikation unter den Mitarbeitenden.

Vor diesem Hintergrund kommt dem Management des Markenimages, unter anderem durch die digitale, unternehmensgenerierte Markenkommunikation hohe Relevanz zu. Für eine detailliertere Annäherung an das Markenimage wird im Weiteren auf folgende Punkte eingegangen: Assoziationen, Image als Einstellung, Image und Reputation.

\section{Assoziationen}

Keller (1993) definiert das Markenimage als «the set of associations linked to the brand that consumer hold im memory» (S. 2). In gleicher Weise bilden nach D. A. Aaker und Joachimsthaler (2000) die «gegenwärtigen Assoziationen» (S. 40) einer Marke deren Markenimage. Die Assoziationen können vielfältige Ausprägungen annehmen und sich auf Produktattribute (z. B. Leistungsinformation, Verpackungsgestaltung), den Produktnutzen (z. B. Fortbewegung, Selbstdarstellung) oder die Marke generell (z. B. mag ich, handelt sozial verantwortlich) beziehen (Henderson, Iacobucci \& Calder, 1998, S. 307; Keller, 1993, S. 34). Diese Definition des Markenimages basiert auf der Schema-Theorie, wonach das Wissen des Menschen in einem semantischen Netzwerk oder Schema gespeichert ist (Bonfadelli \& Friemel, 2011, S. 197). Das Netzwerk besteht aus Knoten (Assoziationen) und Verbindungen zwischen den Knoten (siehe Abbildung 2.2). Durch die Aktivierung eines Knotens (z. B. Markenname) werden über die Verbindungen auch die angrenzenden Knoten (z. B. Eigenschaften dieser Marke) aktiviert (Henderson et al., 1998).

Personen bauen die Assoziationen über den Lauf der Zeit durch direkte Erlebnisse mit der Marke oder über Kommunikation auf. Dahlen, Rosengren, Törn und Öhman (2008) verstehen das Schema als eine «Investition»: «It is the end result of all previous processing that a consumer has devoted to the brand [...]» (S. 58). 


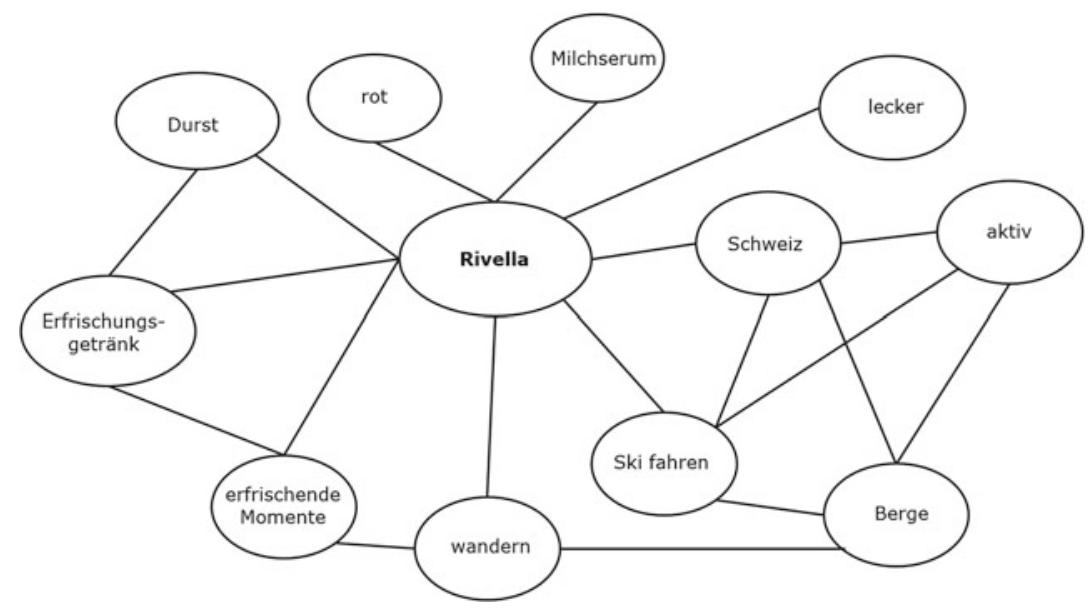

Abbildung 2.2 Beispielhaftes semantisches Netzwerk mit Assoziationen zur Marke Rivella. (Eigene Darstellung)

Schemata helfen dabei als «Wahrnehmungs-, Verarbeitungs- oder Speicherroutinen» (S. 197) bei der Komplexitätsreduktion (Bonfadelli \& Friemel, 2011). Bei der Informationsverarbeitung werden nicht alle objektbezogenen Daten gespeichert, sondern nur die «zentralen und diskriminierenden»(S. 197) Attribute (Bonfadelli \& Friemel, 2011). Im Idealfall sind aus Sicht des Markeninhabers die mit der Marke verbundenen Assoziationen stark, positiv und einzigartig (Keller, 1993, 2013). Je enger die Assoziationen untereinander in Beziehung stehen, desto stärker ist das Markenimage, weil sich Konsumierende dann ein übergreifendes, klares Bild der Marke machen können (Keller, 1993, S. 8). Generell verfügen starke Marken über eine grössere Anzahl an eigenständigen, differenzierenden Assoziationen (Esch, Langner, Schmitt \& Geus, 2006). Das Markenschema, also die mit der Marke verbundenen Assoziationen, unterscheidet sich jedoch von Person zu Person. Auch hier gilt es allerdings Konsistenz anzustreben. Je konsistenter Assoziationen personenübergreifend sind, desto einheitlicher wird eine Marke wahrgenommen und desto stärker ist ihr Image (Rosenbaum-Elliott, Percy \& Pervan, 2011, S. 150). Zur Messung der Assoziationen werden oft qualitative Methoden eingesetzt (Zenker, 2014). 


\section{Image als Einstellung}

Oftmals wird das Markenimage als Einstellung gegenüber der Marke konzipiert. Die beiden Begriffe Image und Einstellung werden dann synonym verwendet (Becker, 2012, S. 66; Kroeber-Riel \& Gröppel-Klein, 2013, S. 233).

Der Einstellungsbegriff in der Markenwissenschaft geht auf die Arbeiten von Fishbein und Ajzen zurück (Burmann \& Stolle, 2007). Diese verstehen Einstellung als «a learned predisposition to respond in a consistently favorable or unfavorable manner with respect to a given object» (Fishbein \& Ajzen, 1975, S. 6). In ihrem «Erwartungswert-Modell» ist eine Einstellung demnach eine affektive (positive oder negative) Einschätzung (S. 11) eines Bezugsobjekts, die sich als die Summe kognitiv gebildeter Auffassungen (beliefs) ergibt (Fishbein \& Ajzen, 1975, S. 12-14). Auffassungen wiederum entstehen, indem ein Objekt (z. B. Schokolade von Lindt) mit einer gewissen, individuellen Wahrscheinlichkeit mit einem Attribut (z. B. hat hohen Kakaogehalt) verbunden wird (S. 12). Attribute werden einer individuellen (affektiven) Bewertung unterzogen (S. 14). Das Attribut «hoher Kakaogehalt» kann sowohl positiv als auch negativ bewertet werden - mit entsprechenden Konsequenzen für die Einstellung und das Verhalten gegenüber der Lindt-Schokolade.

Das Modell von Fishbein und Ajzen repräsentiert ein traditionelles Verständnis des Einstellungsbegriffs, bei welchem die Valenz - die Einschätzung auf dem Kontinuum positiv versus negativ - im Vordergrund steht (Petty, Fabrigar \& Wegener, 2003, S. 752-753). Für Meffert, Burmann, Kirchgeorg und Eisenbeiss (2019) ist das Markenimage hingegen «ein mehrdimensionales Einstellungskonstrukt [eigene Hervorhebung], welches das in der Psyche relevanter externer Zielgruppen fest verankerte, verdichtete und wertende Vorstellungsbild von einer Marke wiedergibt» (S. 268). Diese Mehrdimensionalität des Einstellungsbegriffs kommt in der Zwei- bzw. Drei-Komponenten-Theorie zum Ausdruck. Gemäss der Drei-Komponenten-Theorie besteht eine Einstellung aus einer affektiven, einer kognitiven und einer konativen Komponente (Petty et al., 2003; Wirth \& Kühne, 2013, S. 315). Die konative Komponente - das auf das Einstellungsobjekt bezogene Verhalten - wird jedoch oft vom Einstellungsbegriff ausgekoppelt. Die Einstellung besteht dann im Sinne einer Zwei-Komponenten-Theorie aus einer kognitiven und einer affektiven Komponente (L. Fiedler, Becker \& Kirchgeorg, 2009), gilt aber als Einflussgrösse auf die Verhaltensabsicht (Becker, 2012, S. 69).

Das Markenimage wird im Rahmen dieser Arbeit an die Zwei-KomponentenTheorie angelehnt. Es besteht in diesem Fall aus einer kognitiv-funktionalen und einer affektiv-emotionalen Komponente. Die Operationalisierung dieser beiden Komponenten erfolgt über die beiden grundlegenden Produktnutzen und die damit verbundenen Konsumbedürfnisse (Batra \& Ahtola, 1991). Rossiter und 
Percy (1998) schreiben diesbezüglich: «Brand Attitude is defined as the buyer's evaluation of the brand with respect to its perceived ability to meet a currently relevant motivation» (S. 120). Die kognitive Einstellungskomponente umfasst den funktionalen Produktnutzen (z. B. Rechnungsleistung eines Computers) und das damit verbundene utilitaristische Kundenbedürfnis (z. B. eine Excel-Rechnung erledigen). Die affektive Einstellungskomponente deckt einen emotionalen Produktnutzen (z. B. ein Rivella zu trinken macht Spass) und ein damit verbundenes hedonistisches Konsumbedürfnis (z. B. sich eine gute Zeit gönnen am Feierabend) ab (L. Fiedler et al., 2009; Petty et al., 2003). Das affektiv-emotionale Image deckt damit mannigfaltige mit der Markennutzung verbundene Gefühle ab und bezieht sich nicht länger nur auf die Valenz (siehe Petty et al., 2003, S. 753).

Hirschman und Holbrook (1982) trugen mit der Idee des hedonistischen Konsums bedeutend zur Verbreitung eines zweidimensionalen Markeneinstellungsbegriffs bei (Voss et al., 2003, S. 310). Sie beschreiben den hedonistischen Konsum als «those facets of consumer behavior that relate to the multisensory, fantasy and emotive aspects of product usage experience» (1982, S. 92). Die Autoren sehen den hedonistischen, emotionalen Ansatz als Ergänzung zum traditionellen, kognitiven Ansatz. Während beim hedonistischen Ansatz die mit dem Produkt und dessen Gebrauch verbundenen Emotionen im Vordergrund stehen, geht es beim traditionellen Ansatz um die Funktionalität von Produkten und deren Attribute.

Using a hedonic consumption perspective, products are viewed not as objective entities
but rather as subjective symbols. The researcher is concerned not so much with what
the product is as with what it represents. Product image, not strict reality, is a central
focus; consumer emotive response, rather than just semantic learning, is a key criterion.
Thus, the hedonic perspective seeks not to replace traditional theories of consumption
but rather to extend and enhance their applicability. (Hirschman \& Holbrook, 1982,
S. 93)

In Wissenschaft und Praxis kommt der Zweidimensionalität grosse Bedeutung zu. Batra und Ahtola (1991) beispielsweise lassen Produkte und Marken auf einer hedonistischen (hedonic) und einer funktionalen (utilitarian) Dimension beurteilen. Bei der hedonistischen Dimension stehen das Vergnügen und der Spass der Produktnutzung im Vordergrund, bei der funktionalen Dimension hingegen die Funktionalität des Produkts und seine Zweckorientiertheit. Die Autoren zeigen, dass bei Produkten und Dienstleistungen, die Spass machen (z. B. Rock-Konzert), die hedonistische Dimension die Beurteilung prägt, während bei funktionalen Produkten und Dienstleistungen (z. B. zum Zahnarzt gehen) die funktionale Dimension die Beurteilung prägt. 
Auch Bruhn et al. (2012), Freundt (2006), Rosenbaum-Elliott et al. (2011), Voss et al. (2003) oder Dobni und Zinkhan (1990) unterscheiden zwischen einer kognitiven/utilitaristischen/funktionalen und einer emotionalen/affektiven/hedonistischen Imagedimension. Voss et al. (2003) haben auf Basis von Batra und Ahtola (1991) ein weiterentwickeltes Messmodell vorgeschlagen, anhand dessen sowohl die allgemeine Wahrnehmung von Produkten - einleitend zu diesem Kapitel wurde dazu der von Schmid und Lyczek (2008) gebrauchte Begriff des «generischen Images» (S. 53) genannt - als auch die Wahrnehmung von Marken auf Basis ihres primären Nutzens kategorisiert werden. Diese Unterscheidung ist wichtig, weil das Markenimage nicht mit der Wahrnehmung des generischen Produktimages übereinstimmen muss. Das generische Image des Produkts «Schuh» mag beispielsweise sehr funktional geprägt sein (z. B. Schuhe dienen der Fortbewegung und schützen den Fuss), während das Markenimage von Adidas-Schuhen viel stärker emotional aufgeladen ist.

Abbildung 2.3 zeigt beispielhaft den wahrgenommenen funktionalen und hedonistischen oder emotionalen Nutzen von Produkten (im Sinne eines generischen Images) und von Marken.

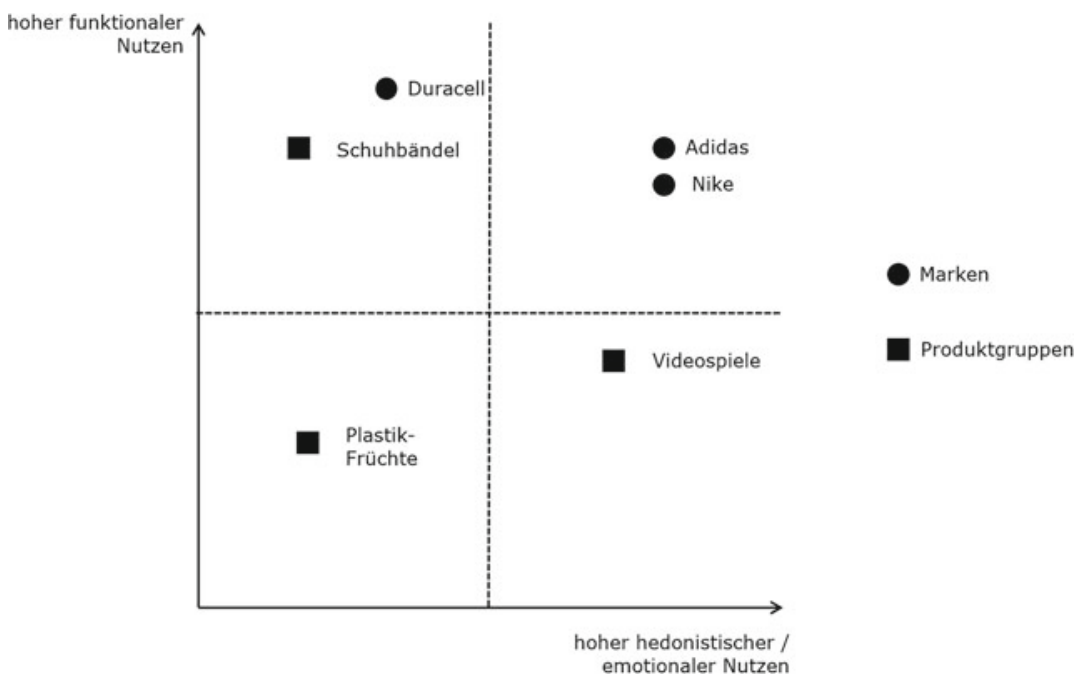

Abbildung 2.3 Marken und Produkte nach funktionalem und emotionalem Nutzen. (Vereinfachte Darstellung auf Basis von Voss, Spangenberg und Grohmann (2003)) 
Auch andere Forschende haben sich mit ähnlichen Produkt- und Markenklassifikationen beschäftigt. Basierend auf solchen Klassifikationen wurden auch Implikationen für die Markenkommunikation abgeleitet. Auf die entsprechenden Modelle von Vaughn (1986) oder Rossiter, Percy und Donovan (1991) wird in Abschnitt 2.3.5 eingegangen.

Die hohe Erklärungskraft eines zweidimensionalen Klassifikationsrasters wurde zudem nicht nur für Marken, sondern für Objekte generell dargelegt. Im Stereotype Content Model (Fiske, Cuddy, Glick \& Xu, 2002) werden Personen oder Objekte auf den beiden Dimensionen Kompetenz und Wärme eingeschätzt. Wärme umschreibt dabei, inwiefern Menschen (in Bezug auf ihre Handlungen) positive oder negative Absichten zugeschrieben werden, während sich Kompetenz darauf bezieht, inwiefern den Menschen zugetraut wird, ihre Absichten in die Tat umzusetzen (Fiske, Cuddy \& Glick, 2007). Auch dieses Modell findet Anwendung in der Markenforschung (J. L. Aaker, Vohs \& Mogilner, 2010; Peter \& Ponzi, 2018).

Die zweidimensionale, auf dem Produktnutzen basierende Operationalisierung des Markenimages wird schliesslich oft um eine nachgelagerte Einschätzung des Globalimages ergänzt. Dieses misst die übergreifende Einstellung gegenüber der Marke (Bruhn et al., 2012; Burmann \& Stolle, 2007; Eilers, 2014; Horstmann, 2017). Eine solche gesamtheitliche, den Nutzen nachgelagerte Einschätzung schlagen auch Rossiter und Percy (1998) vor. Nach ihnen besteht die Markeneinstellung aus vier Bausteinen, wobei sich der zweite Baustein aus zwei Subkomponenten zusammensetzt: (1) eine übergeordnete Auffassung der Marke (superbelief), (2) zwei nutzenbezogene Auffassungen (beliefs), wobei sich die eine Auffassung auf den informativen Nutzen und die andere Auffassung auf den transformativen (emotionalen) Nutzen bezieht, (3) freistehende Emotionen, (4) eine Wahlroute (choice route), anhand welcher der Konsument die nutzenbezogenen Auffassungen und die freistehenden Emotionen zum «Superbelief» zusammenführt.

Daneben gibt es aber auch Operationalisierungen des Markenimages, die auf drei oder mehr Produkt- oder Markennutzen beruhen. Ein solcher Vorschlag stammt beispielsweise von Park et al. (1986), die drei Produktnutzen und entsprechend drei Image-Dimensionen unterscheiden: «functional», «symbolic» und «experiential» (S. 136). Später haben Park, Eisingerich und Park (2013, S. 233) die drei Nutzen wie folgt definiert:

- Enticing the Self: Ästhetischer oder hedonistischer Nutzen

- Enabling the Self: Funktionaler (Produkt-)Nutzen

- Enriching the Self: Nutzen zur Präsentation der eigenen (Soll-)Identität 
Forschende der identitätsbasierten Markenführung (Becker, 2012; Burmann \& Stolle, 2007; Eilers, 2014) unterscheiden zwischen utilitaristischem, sozialem, ästhetischem und hedonistischem Markennutzen. Allerdings müssen diese Unterdimensionen teilweise auf Basis empirischer Überprüfungen ihrerseits wieder in zwei übergreifende Kategorien funktionaler und emotionaler Prägung zusammengefasst werden (Eilers, 2014, S. 136).

Einleitend wurde gesagt, dass ein Markenimage aus den mit der Marke verbundenen Assoziationen besteht. Diese Assoziationen können unterschiedlichster Natur sein und sich auf den funktionalen oder emotionalen Nutzen beziehen, aber auch auf die soziale Verantwortung des Unternehmens, die Herkunft des Unternehmens oder auf mit der Marke verbundene Testimonials. In diesem Sinne ist die Reduktion des Imagebegriffs auf die funktionale und emotionale Dimension eine Vereinfachung, die aber insofern sinnvoll ist, weil die beiden Kategorien zumindest aus Kundensicht markenprägend sind (Batra \& Ahtola, 1991).

\section{Image und Reputation}

Marken müssen sich heute nicht nur an den Bedürfnissen des Marktes und der Kunden, sondern auch an den Ansprüchen vielfältiger Anspruchsgruppen orientieren (Ingenhoff, 2018). Das trifft insbesondere auf Unternehmensmarken zu. Vor diesem Hintergrund muss kritisch reflektiert werden, inwiefern sich eine Operationalisierung des Markenimages einzig am Produktnutzen orientieren kann. Ansätze der betriebswirtschaftlichen und kommunikationswissenschaftlichen Reputationsforschung schlagen hierzu eine breitere Palette an Dimensionen vor. Das wird bereits am Reputationsbegriff deutlich, der sich explizit auf Anspruchsgruppen bezieht: Reputation beschreibt die «aggregierte Wahrnehmung [eines Unternehmens oder einer Marke] einer Vielzahl von Stakeholdern» (Ingenhoff, 2007, S. 7). Entsprechend beziehen sich die Konzepte aber auch primär auf Unternehmen und Unternehmensmarken und weniger auf Produktmarken. Für Schwaiger (2006) ist Corporate Reputation Management «eine konsequente Fortsetzung des Brand Managements» (S. 50) auf Stufe Unternehmens- statt Produktmarke.

Das wirtschaftliche und akademische Interesse an Reputation gewann mit dem steigenden Interesse von Anspruchsgruppen an Unternehmensaktivitäten und einer damit einhergehenden kritischen Beurteilung dieser Aktivitäten an Bedeutung (Fombrun, Gardberg \& Sever, 2000). Die Reputationsforschung hat deshalb insbesondere auch dadurch Aufschwung erhalten, um einschätzen und vergleichen zu können, wie Unternehmen in der Gunst der Anspruchsgruppen bzw. der Öffentlichkeit stehen. Entsprechend nähern sich viele Forschende der Reputation 
aus einer messtechnischen und weniger einer theoretischen Perspektive (siehe Fombrun et al., 2000).

Einer der ersten wissenschaftlichen Ansätze zur Messung von Reputation ist der «Reputation Quotient» (Fombrun et al., 2000). Ausgehend von praxisorientierten Messansätzen wie Fortunes «America's Most Admired Companies» wurde eine wissenschaftlich überprüfte Operationalisierung vorgelegt. Diese bestand aus den sechs Dimensionen «Emotional Appeal», «Products \& Services», «Vision and Leadership», «Workplace Environment», «Social and Environmental Responsibility» und «Financial Performance», die jeweils anhand von drei oder vier Indikatoren gemessen wurden. Während «Emotional Appeal» eine eigene emotionale Kategorie bildet, können die restlichen fünf Dimensionen zu einer Kategorie des «Rational Appeal» zusammengefasst werden. Die Reputation ergibt sich über die Einschätzungen dieser Dimensionen durch die verschiedenen Stakeholder. Damit beschreibt Reputation die durch die Stakeholder zugeschriebene Fähigkeit eines Unternehmens, Gutes für die Stakeholder zu tun («provide valued outcomes», Fombrun et al., 2000, S. 243). In diesem Reputationsansatz spielt der Imagebegriff keine Rolle. In früheren Arbeiten im Feld der «Corporate Communication» wurden die Begriffe Image und Reputation oft synonym verwendet (Simcic Brønn, 2013, S. 55).

Nach Schwaiger (2006) legte der Reputation Quotient zu viel Gewicht auf rationale Aspekte und vernachlässigte die emotionale Dimension. Ebenfalls übt Schwaiger Kritik an der in der amerikanischen Literatur gängigen Gleichsetzung von Image und Reputation (Schwaiger, 2006, S. 49). Der von ihm entwickelte Ansatz (Schwaiger, 2004; 2006) unterteilt die Reputation als «Einstellungskonstrukt» (2004, S. 49) in eine kognitive Kompetenz- und eine emotionale Sympathiedimension. Daneben identifiziert Schwaiger (2004) vier Dimensionen, die als unabhängige Variablen die Reputation beeinflussen: Qualität, Performance, Attraktivität und Verantwortung (S. 65-66). Das Image wird von der Reputation abgegrenzt (S. 50), fliesst jedoch ebenfalls nicht weiter in die Reputationsmessung ein.

Eisenegger und Imhof (2007) wiederum ergänzen die von Schwaiger (2004) vorgeschlagene zweidimensionale Operationalisierung von Reputation um eine normative Komponente. Ihr dreidimensionaler Reputationsbegriff unterscheidet in Anlehnung an das Habermas'sche Drei-Welten-Konzept (Habermas, 1984, 84ff) eine funktionale, eine soziale und eine expressive Reputation.

- Die funktionale Reputation umfasst die Fachkompetenz und gibt darüber Auskunft, «wie gut eine Organisation oder Institution dem Zweck dient, für den sie geschaffen wurde» (Eisenegger \& Imhof, 2007, S. 4). 
- Die soziale Reputation handelt von der Legitimität und davon, «inwieweit (...) gesellschaftliche Normen befolgt werden» (Eisenegger \& Imhof, 2007, S. 4).

- Die expressive Reputation geht der Frage nach, welche «emotionale Attraktivität und Wahrhaftigkeit» (S. 5) von einem Unternehmen ausgeht.

Auch Eisenegger und Imhof beschäftigen sich mit der Abgrenzung von Image und Reputation (S. 10). Images sind, so die Autoren, «neutral konnotiert» und nicht positiver oder negativer Art. Reputation hingegen impliziert eine Rangfolge unter zu vergleichenden Unternehmen oder Organisationen. Dadurch ist Reputation an «öffentliche Bekanntheit» gebunden und wird stark über die Medienöffentlichkeit getrieben (S. 11). Die Reputation von Unternehmen erfassen die Autoren inhaltsanalytisch über die Medienberichterstattung, wobei einzelne Aussagen den drei Reputationsdimensionen zugeordnet werden. Während ein Unternehmen oder eine Organisation unterschiedliche Images besitzen kann, ergibt sich die Reputation als deren Summe (Eisenegger \& Imhof, 2007, S. 10). In vergleichbarer Weise schreibt Mast (2015): «Image ist das spontane, intuitive Bild eines Unternehmens bei einzelnen Zielgruppen, während die Reputation eine meist langfristig orientierte, aggregierte Bewertung von Unternehmenseigenschaften und -handlungen in der Öffentlichkeit darstellt» (S. 7).

Von Ingenhoff (2007) stammt der Ansatz «Integrated Reputation Management System»(IReMS). Im Zentrum des Ansatzes steht die Kopplung von Marktforschungsdaten (z. B. Stakeholder-Einschätzungen der Unternehmensreputation) auf der einen Seite und von Medienresonanzanalysen auf der anderen Seite. Ingenhoff versteht die Reputation ebenfalls als Einstellungskonstrukt und unterteilt diese in eine funktional-kognitive, eine sozial-kognitive und eine affektiv-emotionale Dimension (siehe auch Ingenhoff \& Buhmann, 2016; Ingenhoff, 2018). Die Dimensionen bestehen wiederum aus unterschiedlichen Unterdimensionen:

- Funktional-kognitive Dimension: Produkt- und Dienstleistungsqualität, wirtschaftlicher Erfolg, Managementqualitäten, Kompetenz der Führungspersönlichkeiten, Innovationsfähigkeit und nationale Bedeutung

- Sozial-kognitive Dimension: Übernahme sozialer Verantwortung, Nachhaltigkeit, Sorge um das Mitarbeiterwohl

- Affektiv-emotionale Dimension: Sympathie und Faszination (Ingenhoff, 2007). 
Die Unterdimensionen der funktional-kognitiven und der sozial-kognitiven Dimension stehen in einem formativen Verhältnis zur «Dimension», die affektivemotionale Dimension hingegen wird reflektiv gemessen (siehe Abschnitt 6.3 $\mathrm{zu}$ formativen und reflektiven Indikatoren). Alle Unterdimensionen bestehen aus messbaren Items (Ingenhoff, 2007, S. 58). Die beiden kognitiven Dimensionen wirken sich schliesslich auf die affektiv-emotionale Dimension aus. Je nach Stakeholder-Gruppe wird die Reputation durch unterschiedliche (Sub-) Dimensionen (unterschiedlich stark) getrieben. Entsprechend unterscheidet sich die Reputation eines Unternehmens je nach Gruppe. Diese Einschätzung findet sich auch bei Wiedman, für den die Reputation eine «hoch aggregierte Grösse» ist, weil sie «alle» stakeholderspezifischen Einschätzungen umfasst (Wiedmann, 2012, S. 59). Dennoch kann die Reputation eines Unternehmens «komplex» und «diffus» anstatt «klar» und «prägnant» sein (Wiedmann, 2012, S. 59). Das ist dann der Fall, wenn die Reputation je nach Stakeholder-Gruppe auf unterschiedlich gewichteten Reputationsdimensionen basiert und sich auch die Einschätzung der Dimensionen unterscheidet. Damit unterscheidet sich die Begriffsbestimmung von derjenigen von Eisenegger und Imhof (2007), die Reputation als ausdifferenziertes Resultat der Medienöffentlichkeit verstehen.

Bei der Beschreibung des IReMS geht Ingenhoff nicht explizit auf den Imagebegriff ein, übernimmt jedoch später (Ingenhoff \& Fuhrer, 2010, S. 85) Balmers (2001) Definition von Image als «immediate mental perception of the organization held by an individual, group or network» (S. 257). Reputation ist dann die Kombination unterschiedlicher, durch Stakeholder gehaltener Images (Ingenhoff \& Fuhrer, 2010, S. 85; Ingenhoff, 2018, S. 4). Dieser Beschreibung nach ergibt sich Reputation nun als übergeordnete Grösse auf Basis der durch die Stakeholder gehaltenen Images. Eine solche Begriffsbestimmung, wonach Image eine vergleichsweise dynamischere, einzelnen Individuen oder Gruppen innewohnende Einschätzung und die Reputation eine vergleichsweise stabilere, aggregierte Einschätzung eines Bezugsobjekts ist, findet sich auch bei anderen Autoren (Niederhäuser \& Rosenberger, 2017, S. 12; Thiessen, 2011, S. 31).

Die Abgrenzung von Reputation und Image geschieht bei Wiedmann über die Zeitvariable: «die Reputation eines Unternehmens geht insofern über dessen Image hinaus, als sich hierin zugleich die in der Vergangenheit aufgebauten und für die Zukunft relevanten Unterstützungspotenziale manifestieren» (Wiedmann, 2012, S. 59). Unter Unterstützungspotenzial können dabei beispielsweise Kaufabsicht, Weiterempfehlungsabsicht oder Verteidigung bei Kritik angesehen werden. Das Ziel der Reputation liegt also nicht im «Vermitteln positiver Eindrücke» (S. 60), sondern im Aufbau solcher Unterstützungspotenziale (Wiedmann, 2012). 
Die Überlegungen Wiedmanns beziehen sich auf das Messmodell RepTrak, das eine Weiterentwicklung des Reputation Quotient darstellt (Wiedmann, Fombrun \& van Riel, 2006; Wiedmann, 2012). Die Reputation wird dabei sowohl über formative Reputationstreiber als auch über reflektive Reputationsreflektoren gemessen (Wiedmann, 2012, S. 61-62). Insgesamt werden sieben Reputationstreiber (Performance, Product, Innovation, Workplace, Governance, Citizenship, Leadership) und vier Reputationsreflektoren erhoben (Esteem, Admire, Trust, Feeling).

Zusammenfassend lassen sich folgende Hinweise für die Operationalisierung des Markenimages aus der Reputationsliteratur ziehen. Die Reputation wird wie auch das Markenimage als Einstellungskonstrukt konzipiert (Ingenhoff \& Buhmann, 2016). Beide Konstrukte dienen unter anderem der Differenzierung im Markt (Ingenhoff, 2007, S. 55). Reputation ist je nach Auffassung eine «aggregierte» und einzigartige Grösse, die sich aus den einzelnen, stakeholderspezifischen Images ergibt, oder aber die pro Anspruchsgruppe «aggregierte» Einschätzung eines Unternehmens. Der ersten Auffassung nach kann ein Unternehmen nur eine Reputation, dafür verschiedene stakeholderbezogene Images haben. Der zweiten Auffassung nach hat ein Unternehmen verschiedene Reputationen bei verschiedenen Stakeholdern. Im Gegensatz zum nutzenorientierten Verständnis von Markenimage basiert die Messung der Reputation auf einem breiteren Feld an Einflussfaktoren und Indikatoren und berücksichtigt Elemente wie das Management, die Rolle des Unternehmens als Arbeitgeber oder dessen gelebte Nachhaltigkeit. Diese Vielfältigkeit zeigt sich etwa in Ingenhoffs IReMS, in dem Reputation dreidimensional gemessen wird und eine funktional-kognitive, sozial-kognitive und affektiv-emotionale Dimensionen umfasst (Ingenhoff, 2007). Insbesondere die sozial-kognitive Dimension, die misst, inwiefern ein Unternehmen ein für die Gesellschaft «gutes» Unternehmen ist, bleibt bei der nutzenorientierten Markenimagemessung unberücksichtigt.

Da Corporate Reputation Management «die konsequente Fortsetzung des Brand Managements» (S. 50) auf Stufe Unternehmens- statt Produktmarke ist (Schwaiger, 2006) und eine breitere Palette an Imagedimensionen vorschlägt als die Operationalisierung des Markenimages über den Produktnutzen, lässt sich festhalten: Je näher sich eine Marke auf dem Kontinuum Produktmarke versus Unternehmensmarke hin zur Unternehmensmarke bewegt, desto relevanter wird sie über die marktorientierte Zielgruppe hinweg für ein breites Spektrum an Anspruchsgruppen und desto stärker nähert sich das Markenimage der Unternehmensreputation an. 
Im Rahmen dieser Arbeit wird im Folgenden der Begriff Markenimage (statt Reputation) verwendet. Damit ist das Vorstellungsbild der Marke oder eines markierten Produkts im Sinne der mit der Marke verbundenen Assoziationen gemeint. Allerdings wird - in einer vereinfachten, reduzierten Weise - der Fokus auf das funktional-kognitive und das emotional-affektive Markenimage sowie auf das Globalimage der Marke gelegt. Diese beiden Imagedimensionen sind aus einer Konsumentensicht prägend (Voss et al., 2003) und spielen auch in der Reputationsforschung eine zentrale Rolle (Ingenhoff, 2007; Ingenhoff \& Buhmann, 2016; Ingenhoff, 2018). Das Markenimage kann sich auf eine spezifische Gruppe (z. B. Kundinnen und Kunden, Follower der Marke auf Facebook, Mitglieder einer Community) oder auf die Gesamtheit aller Anspruchsgruppen beziehen. Gerade starke Marken zeichnen sich dadurch aus, dass sie positive und einzigartige Assoziationen über alle Anspruchsgruppen hinweg etablieren können.

\subsubsection{Markenpositionierung}

Der Aufbau eines (starken) Markenimages ist ein langfristiger Prozess, dem sich die Positionierung widmet. Die Markenpositionierung hat zum Ziel, eine Marke in den Köpfen der Ziel- oder Anspruchsgruppen zu verankern. Verankern heisst in diesem Kontext, dass die Marke mit wenigen ausgewählten Assoziationen in Verbindung gebracht werden soll (Esch, 2012, S. 90). Diese ausgewählten Assoziationen (z. B. «Freude am Fahren» bei BMW) repräsentieren die in der Identität verankerten «Stärken» (Niederhäuser \& Rosenberger, 2017, S. 36) oder Nutzen der Marke und sorgen gleichzeitig für Differenzierung gegenüber der Konkurrenz (D. A. Aaker \& Joachimsthaler, 2000, S. 41-42). Die Auswahl der Assoziationen orientiert sich traditionell am Produktnutzen und dem dazugehörenden Kundenbedürfnis (Schmid \& Lyczek, 2008, S. 88). Im vorherigen Kapitel wurde diesbezüglich dargelegt, dass die relevantesten Assoziationen aus Kundensicht den funktionalen oder emotionalen Markennutzen abbilden. Die Markenpositionierung ist also ein strategisches Instrument des Unternehmens, das sich mit der Verankerung von Assoziationen bzw. eines idealen funktionalen oder emotionalen Soll-Images aus Unternehmenssicht befasst. Die Positionierung ist eng an die Markenidentität angelehnt (D. A. Aaker \& Joachimsthaler, 2000, S. 41). In Abbildung 2.1 ist deshalb die Markenpositionierung gänzlich im unternehmensinternen Raum verortet. Die Positionierung kommt damit gewissermassen dem Versprechen gleich, das die Marke gegenüber den Zielund Anspruchsgruppen abgibt. Die Positionierung legt den Grundstein für die 
Kommunikationsarbeit. Positionierung kann in diesem Sinne auch als eine «übergeordnete Kommunikationsstrategie» (Esch \& Honal, 2018, S. 4) verstanden werden. ${ }^{5}$

In der Frühzeit des Markenwesens reichte es, ein Produkt und dessen funktionalen Nutzen bekannt zu machen (Merz, He \& Vargo, 2009). Eine Seife wurde gekauft, um sich zu reinigen, ein Auto diente der Mobilität und eine warme Jacke schützte vor Kälte. Die Positionierung beschränkte sich darauf, Assoziationen über ein mehr oder weniger umfassendes Set an Grundfunktionen aufzubauen. Im Laufe der Zeit hat sich die Marke von einem Träger eines funktionalen Nutzens hin zu einem Träger eines symbolischen oder emotionalen Nutzens entwickelt (Merz et al., 2009). Damit trug die Marke selbst zur Wertschöpfung bei und ergänzte den rein funktionalen Produktnutzen, den die Marke ursprünglich auszuweisen hatte (siehe Hellmann, 2003, S. 77). Laut Esch (2016) zeichnen sich gerade starke Marken dadurch aus, dass die Markenassoziationen über die spezifischen Vorstellungen zur Produktkategorie hinausgehen; dies auch vor dem Hintergrund, weil «Vorstellungen zu einer Produktkategorie [...] automatisch auf jede Marke der Kategorie übertragen» (S. 26) werden. Auch D. A. Aaker, Stahl und Stöckle (2015, S. 50) betonen die Wichtigkeit, mehr als den funktionalen Nutzen hervorzuheben. Dies, weil Kunden nicht rational handeln, sondern emotionale, soziale oder selbstdarstellende Nutzen in die Entscheidung einfliessen lassen. Die Positionierung hat dadurch zunehmend zum Ziel, «an sich austauschbaren Produkten Eigenständigkeit zu verleihen»(Kloss, 2012, S. 119), anstatt die Funktionalität des Produkts zu vermitteln.

Eine Positionierung auf Basis eines Zusatznutzens kann viele verschiedene Ausprägungen annehmen. Ein Produkt kann emotional aufgeladen werden, mit bekannten Persönlichkeiten beworben werden, sich Assoziationen des Herkunftslandes zugute machen oder eine nachhaltige Produktion in den Vordergrund stellen. Prinzipiell können sämtliche Dimensionen und Treiber von Image und

\footnotetext{
${ }^{5}$ Mit Positionierung wird teilweise auch die tatsächliche «Position» einer Marke in den Augen der Zielgruppen beschrieben. Dadurch rückt Positionierung in die Nähe des Markenimages (Burmann et al., 2018, S. 102). Im empirischen Teil dieser Arbeit wird Markenpositionierung in dieser Weise verwendet. Als Teil der experimentellen Manipulation wird unterschieden, ob eine Marke gemäss ihrer Positionierung primär aus rationalen/kopforientierten Gründen gekauft wird und damit primär ein funktionales Bedürfnis befriedigt oder ob eine Marke primär aus emotionalen/gefühlsorientierten Gründen gekauft wird und damit ein emotionales Bedürfnis befriedigt (K. Klein \& Melnyk, 2016, S. 126). Grundsätzlich könnte diese Frage auch als ein Indikator des bestehenden Markenimages aufgefasst werden. Auf den Begriff Markenimage wurde in diesem Zusammenhang allerdings bewusst verzichtet, damit dieser der abhängigen Zielvariable vorbehalten bleibt.
} 
Reputation, wie sie im vorhergehenden Kapitel vorgestellt wurden, als Positionierungsgrundlage dienen.

Freundt (2006) sieht in der «Qualitätsnivellierung» (S. 10) und der damit einhergehenden, zunehmenden Austauschbarkeit von Produkten einen Grund für die sich verändernde Funktion der Marke. In vergleichbarer Weise macht Baumgartner (2009) die hohe Marktsättigung und die damit verbundene Austauschbarkeit der Produkte als Wegbereiter für «immaterielle» und «sinnstiftende» (S. 33) Zusatznutzen aus. Eine attraktive Differenzierung im Markt über den funktionalen Produktnutzen ist schwierig, weil Produktinnovationen, wie sie beispielsweise Apple ab Mitte der 2000er-Jahre einführte (MacBook Air, Smartphones, Tablets), oft innerhalb kurzer Zeit durch die Konkurrenz kopiert werden. Entsprechend rücken bei der Positionierung zunehmend emotionale Zusatznutzen in den Fokus, die den funktionalen Grundnutzen ergänzen (Kloss, 2012, S. 120). Die zunehmende Relevanz eines emotionalen Zusatznutzens darf allerdings nicht darüber hinwegtäuschen, dass sich viele starke Marken nach wie vor auch oder gerade durch den funktionalen Basisnutzen im Markt differenzieren - selbst wenn ein emotionaler Zusatznutzen mitspielt. Hohe Funktionalität bei alpiner Kleidung (Mammut), langanhaltender Sonnenschutz (Daylong) oder hohe Einsatzbereitschaft und Zuverlässigkeit bei Schienenfahrzeugen (Stadler Rail) sind funktionale Nutzen, die die Basis erfolgreicher Marken bilden.

Der Erfolg einer prägnanten Positionierung liegt in der Reduktion, weil nur wenige Attribute oder Assoziationen langfristig glaubhaft vermittelt und in den Köpfen der Zielgruppen nachhaltig besetzt werden können (Langner, Brune, Fischer \& Klinke, 2018, S. 27). Höchstens drei positive Vorurteile vermögen starke Marken, so Zschiesche und Errichiello (2015, S. 17), in den Köpfen zu verankern. Und dies geschieht nur durch regelmässige Wiederholung. Entsprechend ist es bedeutend, dass Marken über lange Zeit gleichbleibende Leistungen erfüllen und kommunizieren und somit «Vertrauen in die Leistung» (S. 17) entsteht (Zschiesche \& Errichiello, 2015).

\subsubsection{Klassische Markenkommunikation}

Wenn Positionierung die «übergeordnete Kommunikationsstrategie» (Esch \& Honal, 2018, S. 4) ist, dann kann die klassische Markenkommunikation als die operative Umsetzung dieser Strategie verstanden werden. Die klassische Markenkommunikation hat die Etablierung eines intendierten funktionalen oder emotionalen Markenimages zum Ziel (Esch, 2019). Diese klassische Sichtweise 
wird im weiteren Verlauf als Unternehmensperspektive der Markenkommunikation bezeichnet. Ein zentrales Element davon ist «Werbung», also die Schaltung persuasiver Botschaften in bezahlten Kanälen, um eine positive Einstellung gegenüber einem Produkt oder Objekt zu erzielen (Siegert, Wirth, Lischka \& Weber, 2016). Viele Ausführungen dieses Kapitels beziehen sich deswegen auf Werbung.

\section{Informative und emotionale Markenkommunikation (Unternehmensperspektive)}

Im vorherigen Kapitel wurde gezeigt, dass mit einer Positionierung spezifische Assoziationen der Marke in den Fokus gerückt werden und ein ideales Markenimage aufgebaut werden soll. Diese Assoziationen beziehen sich oftmals auf den funktionalen oder emotionalen Markennutzen. Die Dichotomie zwischen funktionalen und emotionalen Aspekten bei Markenimage und Positionierung spiegelt sich konsequenterweise auch in der Kommunikationsarbeit wider. Die Markenkommunikation richtet sich an der Positionierung aus (Langner et al., 2018, S. 27). Entsprechend kann auch bezüglich Kommunikation zwischen zwei grundlegenden Strategien unterschieden werden: «Despite an array of terminology there is general agreement that there are two types of message appeal» (Mortimer, 2008, S. 104).

Zur Benennung dieser beiden Strategien wurden viele verschiedene Begriffe verwendet. Puto und Wells (1984) und später weitere Autoren (B. D. Cutler, Thomas \& Rao, 2000; E. Kim, Lin \& Sung, 2013; Laskey, Day \& Crask, 1989) unterscheiden zwischen informationaler und transformationaler Werbung. Die informationale Werbestrategie zielt darauf ab, Konsumierende mit faktischen, überprüfbaren Informationen über Produkte und Marken zu versorgen, damit diese die Qualität von Produkt und Marke einschätzen können. Die transformationale Werbestrategie hingegen reichert das mit der Produktnutzung verbundene Erlebnis an und macht dieses «reicher, wärmer, spannender und angenehmer» (Puto \& Wells, 1984, S. 638). Van den Putte und Dhondt (2005, S. 2401) sprechen in ähnlicher Hinsicht von den emotionalen Konsequenzen einer Produktnutzung, die bei einer emotionalen Kommunikationsstrategie herausgestrichen werden.

Bei Johar und Sirgy (1991) findet sich in der Tradition des transformationalen Ansatzes der «value-expressive», symbolische oder Image-Ansatz. Mit diesem Ansatz soll das Image «des prototypischen Produktnutzenden» (S. 23) gezeichnet werden (Johar \& Sirgy, 1991). Auf der anderen Seite findet sich in der Tradition des informationalen Ansatzes der utilitaristische, funktionale Ansatz. Dieser Ansatz streicht die Funktionalität von Produkt oder Marke heraus. In vergleichbarer Manier unterscheidet Meenaghan (1995) zwischen der kognitiven und der Brand-Image-Schule der Werbewirkung. Kroeber-Riel und Weinberg 
(2003, S. 616) trennen schlicht zwischen informativer und emotionaler Werbung. Bei der informativen Strategie stehen «sachliche Informationen» über das Produkt oder über das Unternehmen im Vordergrund. Bei der emotionalen Werbung stehen «emotionale Reize» im Zentrum. Insbesondere wird auch Humor als Gestaltungsmerkmal emotionaler Kommunikation verstanden (Kroeber-Riel \& Gröppel-Klein, 2019, S. 561-563).

Bei Okazaki, Mueller und Taylor (2010) finden sich die Begriffe «Soft Sell» und «Hard Sell». Beide Ansätze weisen in ihren Definitionen grosse Parallelen zu den bereits erwähnten Konzeptionen auf. Der Hard-Sell-Ansatz zielt durch die Vermittlung von Fakten und Informationen auf eine rationale Reaktion des Konsumierenden. Der Soft-Sell-Ansatz nutzt emotionale Geschichten, baut «Image und Atmosphäre» auf und hat eine affektive Reaktion des Konsumierenden zum Ziel. Im Gegensatz zu den bisher zitierten Ansätzen wird bei beiden Ansätzen auch eine Aussage über die Mittelbarkeit der Wirkung getroffen. Der Hard-SellAnsatz wirkt direkt und soll explizit zum Kauf animieren, der Soft-Sell-Ansatz wirkt indirekt und implizit. Mit den Worten Durgees (1988) lässt sich die aufgezeigte Dichotomie der Marketingkommunikation wie folgt zusammenfassen: «Advertising has two purposes: to excite and to inform» (S. 21).

\section{Ausrichten der Markenkommunikation am Produktnutzen}

Werbewirkungsmodelle, z. B. diejenigen von Vaughn (1986) und Rossiter et al. (1991), postulieren, dass die Art der Markenkommunikation am Produktnutzen ausgemacht werden soll für eine möglichst hohe Effektivität. Vaughn (1986) unterscheidet mittels zweier Achsen - «Think-Produkte» und «Feel-Produkte» auf der einen und «High-Involvement-Produkte» und «LowInvolvement-Produkte» auf der anderen - vier verschiedene Quadranten, in welche Produkte eingeordnet werden können (siehe Abbildung 2.4). Involvement beschreibt dabei das Ausmass an Zeit und Ressourcen, das Konsumierende in eine Kaufentscheidung investieren angesichts der Konsequenzen dieser Entscheidung (Vaughn, 1986).

Je nach Quadrant bietet sich eine andere Kommunikationsstrategie an (Vaughn, 1986). Ein Think-Produkt mit hohem Involvement (z. B. Versicherungsprodukt) wird im Idealfall durch eine informative Kommunikationsstrategie unterstützt. Ein Think-Produkt mit tiefem Involvement (z. B. Toilettenpapier) hingegen verlangt nicht mehr als eine habituelle Kommunikation, anhand welcher die Konsumenten an die Existenz des Produkts erinnert werden. Bei einem FeelProdukt mit hohem Involvement (z. B. Premium-Parfum) bietet sich eine affektive (emotionale) Kommunikationsstrategie an, während eine «Satisfaction-Strategie» ideal für Feel-Produkte tiefen Involvements ist. Letztere Strategie zielt darauf ab, 


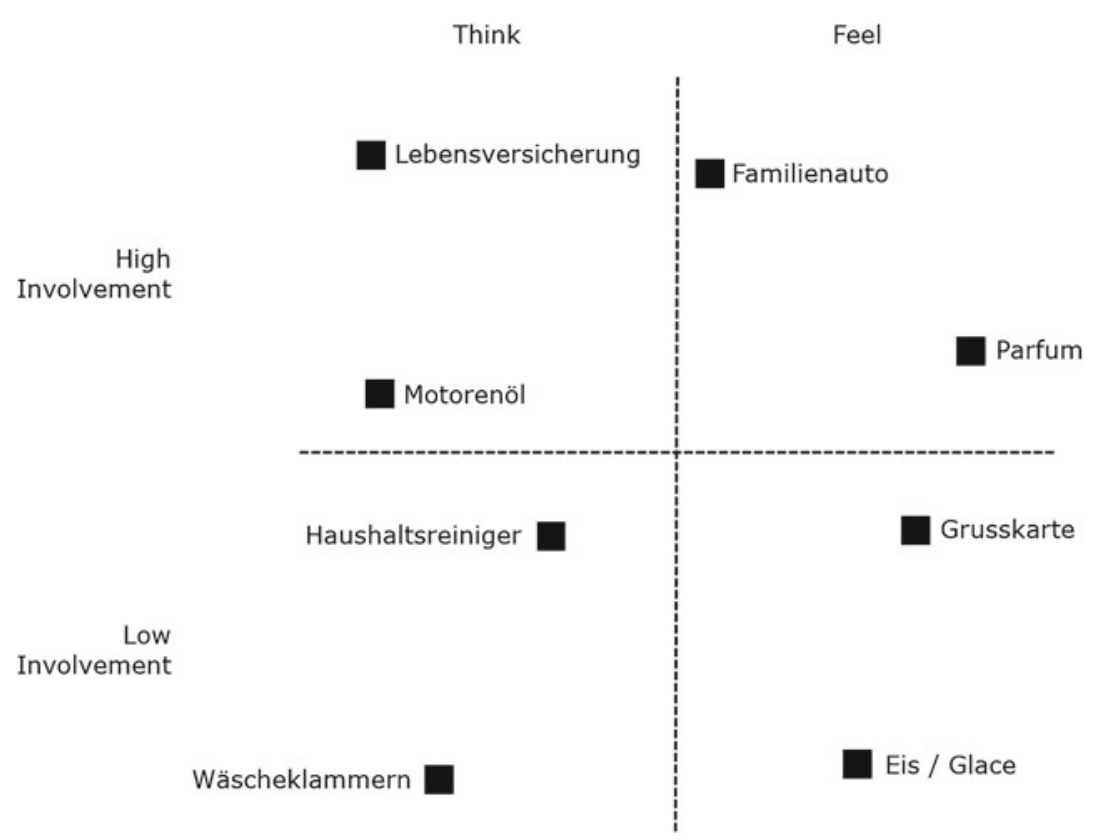

Abbildung 2.4 Verortung von Produkten im FCB-Grid ${ }^{6}$. (Nach Vaughn (1986))

Menschen zum spontanen Kauf zu animieren, indem die sofortige Belohnung durch die Produktnutzung in Aussicht gestellt wird. Das Modell von Rossiter et al. (1991) berücksichtigt zusätzlich die Markenbekanntheit. Im Zentrum stehen allerdings ebenfalls vier Quadranten, aufgeteilt durch eine InvolvementAchse und eine Achse, die zwischen informationalen und transformationalen Kundenbedürfnissen unterscheidet. Je nach Quadrant ergeben sich unterschiedliche Implikationen zur Verwendung informativer und emotionaler Elemente in der Markenbotschaft (Rossiter et al., 1991, S. 18-19).

\section{Ausrichten der Markenkommunikation an einem Soll-Markenimage}

Mit Hinblick auf eine anzustrebende Positionierung und den Aufbau eines differenzierenden, relevanten Markenimages müssen die Empfehlungen dieser Modelle mit Vorsicht betrachtet werden. Geht beispielsweise der durchschnittliche Konsument davon aus, dass in einer Produktkategorie ein gewisser minimaler

\footnotetext{
${ }^{6}$ Nach der Werbeagentur Foote, Cone \& Belding (FCB)
} 
Qualitätsstandard erreicht ist, dann kann es sinnvoll sein, ein ursprünglich funktionales Produkt über einen emotionalen Zusatznutzen und entsprechend über eine emotionale Kommunikation zu positionieren. Die Kommunikation richtet sich dann nicht mehr am Produktnutzen aus, sondern an einem (zukünftigen) Soll-Image (siehe auch Bruhn, 2014, S. 80). Der Aufbau eines starken funktionalen oder emotionalen Markenimages verlangt eine langfristig gleichbleibende, konsistente Markenkommunikation, mit der wenige ausgewählte, in der Positionierungsstrategie festgeschriebene Assoziationen immer und immer wieder mit der Marke in Verbindung gebracht werden (Esch, Brunner \& Petri, 2016; Esch, 2019). Konzepte der integrierten Kommunikation beschäftigen sich mit dieser gleichbleibenden, fokussierten Kommunikation, «um ein für die Zielgruppen der Kommunikation konsistentes Erscheinungsbild des Unternehmens bzw. eines Bezugsobjektes der Kommunikation [z. B. Marke] zu vermitteln» (Bruhn, 2014, S. 38). Nur eine solche, langfristig konsistente Kommunikation erlaubt die Etablierung eines starken Markenimages, das sich in der Konsequenz vom generischen Produktimage unterscheidet.

Im Kern beruht diese klassische, marketingbasierte Markenkommunikation auf einem einseitigen Kommunikationsverständnis, wonach mächtige Sender (Unternehmen) ihre Markenbotschaften an empfängliche Rezipienten (Konsumenten) richten (Rappaport, 2007). Mit der Digitalisierung haben sich die Machtverhältnisse ausgeglichen und Unternehmen und Konsumenten begegnen sich auf Augenhöhe. Vor allem die durch das Web 2.0 neu entstandenen sozialen Medien haben die Möglichkeiten der User-Teilnahme und -Einflussnahme multipliziert (siehe Abschnitt 2.2). Unter diesen Voraussetzungen muss sich auch die Markenkommunikation neu ausrichten. Mit der Markenkommunikation im digitalen Umfeld beschäftigt sich das nächste Kapitel.

\subsection{Markenkommunikation in einer digitalen Kommunikationslandschaft}

In der Einleitung wurde anhand von fünf Entwicklungen dargelegt, wie sich die Kommunikationslandschaft für Marken durch die Digitalisierung verändert hat: mehr Kanäle, mehr Kommunikationsteilnehmer, mehr Inhalte, mehr Selektion, mehr Relevanz. Durch die zunehmende Anzahl an Kommunikationsteilnehmern, Inhalten und Kanälen wird es für Marken zunehmend schwieriger, mit den eigenen Botschaften zu den Zielgruppen durchzudringen. Im digitalen Raum werden nur diejenigen Inhalte betrachtet und verbreitet, die relevant sind. Das Internet ist ein «empfängergesteuertes Medium»(Eilers, 2014, S. 38). Deshalb müssen 
auch Unternehmen ihre Markenbotschaften zunehmend an den Regeln digitaler Kommunikation ausrichten und den Usern einen Mehrwert bieten.

Die Herausforderung dieser dialogorientierten Kommunikationslandschaft liegt darin, die Bedürfnisse der Unternehmens- und der Userseite gleichberechtigt zu behandeln. Traditionell wurden starke Marken durch eine einheitliche, gleichbleibende Kommunikation aufgebaut. Diese Kommunikation wurde durch den Markeninhaber gesteuert und sie nutzte langfristig dieselben Kernbotschaften, um die Marke bei den Zielgruppen bekannt zu machen (Esch, Brunner \& Petri, 2016, S. 130), in deren Köpfen ein gewünschtes Vorstellungsbild (Markenimage) aufzubauen und sie letztlich zum Kauf der Marke zu animieren. Diese Unternehmensperspektive wurde in Abschnitt 2.3.5 beschrieben. Die Userperspektive bildet hingegen die Anforderungen der Kundinnen, Kunden bzw. Userinnen und User an eine digitale Markenkommunikation ab. In der digitalen Welt reicht es nicht mehr, Markeninhalte top-down zu definieren und dabei die Bedürfnisse der Kunden zu ignorieren. Es ist allerdings genauso wenig zielführend, Markeninhalte konsequent an Kundenbedürfnissen auszurichten und damit das eigene Markenimage zu verwässern. Vor diesem Hintergrund muss sich die unternehmensgenerierte Markenkommunikation in zweierlei Hinsicht umorientieren. Sie muss erstens intern breiter gefasst werden, also über eine reine Marketingperspektive hinausgehen (gedankliche Umorientierung), und zweitens einen Mehrwert für den User beinhalten (inhaltliche Umorientierung). Auf diese beiden Veränderungen, die ebenfalls in Abbildung 2.5 dargestellt sind, wird im Folgenden näher eingegangen.

\subsubsection{Gedankliche Umorientierung}

Traditionell, das wurde in Abschnitt 2.3.5 dargelegt, orientierte sich die Markenkommunikation an der Marketingkommunikation bzw. an der Werbung. Die beiden anderen Teilbereiche der Unternehmenskommunikation, die interne Kommunikation und die Public Relations (Tropp, 2014a; Zerfass, 2014), spielten eine untergeordnete Rolle für die Markenkommunikation. Das ergibt sich aus deren traditionellen Rollen: Die interne Kommunikation informiert die Mitarbeiterinnen und Mitarbeiter über Managemententscheide, bietet ihnen Orientierung über die strategischen Ziele des Unternehmens und schafft Angebote, damit sich die Angestellten untereinander austauschen können (Jecker, Albisser \& Boenigk, 2019). Public Relations stellt die Strategiedurchsetzung im gesellschaftspolitischen Umfeld sicher, indem sie Handlungsspielräume und Legitimation für die unternehmerischen Tätigkeiten schafft (Zerfass, 2010, S. 298). 
Klassische

Markenkommunikation

Marketing-
kommu-

kommu-
nikation

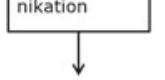

Markenkommunikation als

Marketingkommunikation

(Unternehmensperspektive)

Kundinnen und Kunden als

Zielgruppen der

Markenkommunikation

\section{(1) Gedankliche Umorientierung \\ (2) Inhaltliche Umorientierung}

Markenkommunikation in einer digitalen Kommunikationslandschaft

\section{(a)}

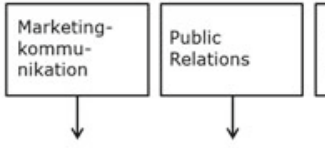

Interne

Kommu-

nikation

Markenkommunikation als Botschaften

der Unternehmenskommunikation

(Unternehmensperspektive)

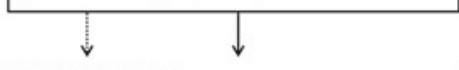

z.B.

traditionelle

Werbung

Brand Content: Kombination der Unternehmens- und Userperspektive

Abbildung 2.5 Von der klassischen zur digitalen Markenkommunikation. (Eigene Darstellung)

Die Verantwortung der Markenkommunikation allein in die Hand des Marketings bzw. der Marketingkommunikation zu legen ist heute allerdings nicht mehr zeitgemäss. Zielführender ist es, Markenbotschaften als Botschaften der Unternehmenskommunikation zu verstehen, welche die drei Teilbereiche Marketingkommunikation, interne Kommunikation und Public Relations umfasst. Sie kann nach Mast (2019), folgendermassen definiert werden kann: «Unternehmenskommunikation (〈Corporate Communications〉) bezieht sich auf die Aufgabe von Organisationen, mit Stakeholdern leistungsfähige Kommunikationsbeziehungen zu unterhalten und zu pflegen» (S. 8).

Aus primär drei Gründen ist dieser Perspektivenwechsel sinnvoll:

Erstens: Der Geltungsanspruch der Marke hat sich vergrössert. Eine Marke muss sich nicht nur am Markt gegenüber den Kunden, sondern zunehmend in einem breiten ökonomischen, sozialen und gesellschaftlichen Umfeld gegenüber einer Vielzahl von Anspruchsgruppen bewähren (Ingenhoff, 2004). Wie eine Marke wahrgenommen wird, hängt dabei von der Gesamtheit aller Interaktionen zwischen dem Unternehmen als Markeninhaber und den Anspruchsgruppen ab. 
Diesem breiten Geltungsanspruch wird die Unternehmenskommunikation glaubwürdiger gerecht als die Marketingkommunikation. Zerfass und Dühring (2016) sehen denn auch im gesellschaftlichen Wandel vom Shareholder-Fokus auf den Stakeholder-Fokus die steigende Bedeutung der Unternehmenskommunikation (siehe auch Bernstein, 2009). Wo der Fortbestand eines Unternehmens nicht länger nur von rein finanziellen Kennzahlen abhängt, sondern von der Einbettung des Unternehmens im gesamten Stakeholder-Umfeld (Zerfass \& Dühring, 2016), ergeben sich neue Implikationen für die Marke. Diese ist nicht länger nur ein Verkaufsinstrument, sondern sichert die «licence to operate» (Süss, Zerfass \& Dühring, 2011, S. 6). Die Public Relations, die traditionell die kommunikativen Beziehungen zu politischen, kulturellen oder ökologischen Anspruchsgruppen koordiniert, trägt heute stark zur Wahrnehmung einer Marke bei (Chung \& Ahn, 2013).

Zweitens: In einem digitalen Kommunikationsumfeld verschmelzen die drei Teilbereiche der Unternehmenskommunikation. ${ }^{7}$ Insbesondere die Public Relations und die Marketingkommunikation können nicht mehr trennscharf voneinander abgegrenzt werden (Ruehl \& Ingenhoff, 2017, S. 170; Zerfass \& Dühring, 2016, S. 60). Damit eine Marke ihren digitalen Zuhörern spannende Inhalte liefern kann, müssen Kommunikationsfachleute unterschiedlicher Fachrichtungen rund um die Uhr zusammenarbeiten und interessante Geschichten aus dem gesamten Unternehmen verarbeiten. Die Geschwindigkeit der Dialoge und Interaktionen in einem digitalen Kommunikationsumfeld erhöht zudem die Notwendigkeit, dass sich alle Kommunikationsfachleute eines Unternehmens im Klaren über die Ausrichtung der Marke (Markenidentität) und deren Kernbotschaften sind. Sie müssen in ihrer täglichen Arbeit rasch und selbstständig auf Kommentare und Fragen in sozialen Medien reagieren, ohne Rücksprache mit Vorgesetzten oder Markenverantwortlichen (Valos, Haji Habibi, Casidy, Driesener \& Maplestone, 2016, S. 30-31). Der Vermittlung der Markenidentität über die interne Kommunikation kommt entsprechend grosse Bedeutung zu (Boenigk, Jecker \& Albisser, 2019). Die interne Kommunikation muss den Kern einer Marke gegenüber den Mitarbeitenden transportieren, damit diese in den Interaktionen mit den Anspruchsgruppen markenkonform handeln können. Die Wahrnehmung eines Unternehmens, eines Produkts oder einer Marke hängt stark vom Verhalten der jeweiligen Mitarbeitenden ab (Chernatony \& Harris, 2000).

\footnotetext{
${ }^{7}$ Die Verschmelzung der drei Teilbereiche ist traditionell bei Unternehmensmarken stärker ausgeprägt, gewinnt jedoch auch bei Produktmarken an Bedeutung (Bernstein, 2009, S. 605; Schmid \& Lyczek, 2008, S. 131; Tropp, 2014a, S. 1102).
} 
Weil die Teilbereiche immer stärker zusammenfliessen (Ruehl \& Ingenhoff, 2017, S. 170), muss integrierte Kommunikation - das Ausrichten der Kommunikation auf eine einheitliche Botschaft - heute nicht mehr nur auf der Ebene der Kommunikationshandlungen, sondern vor allem auf der Ebene des Kommunikationsmanagements geschehen (zu den beiden Varianten der Integration: Zerfass, 2010, S. 311). Während auf der Ebene der Kommunikationshandlungen Botschaften inhaltlich, formal und zeitlich über die und innerhalb der Teilbereiche aufeinander abgestimmt werden (Bruhn, 2014, S. 134), verlangt Integration auf der Ebene des Kommunikationsmanagements, dass «Steuerungspotentiale in allen Managementfunktionen angelegt werden» (Zerfass, 2010, S. 313). Eine Teilaufgabe besteht dann darin, eine Kommunikationsphilosophie und «integrativ wirkende Kernbotschaften» zu entwickeln, die eine «strategische Profilierung» (Zerfass, 2010, S. 313) ermöglichen. Erfolgreiche integrierte Markenkommunikation ist nur zu erreichen, wenn sich diese an einer strategischen Profilierung auf Ebene der Unternehmenskommunikation orientiert.

Drittens: Auch die diversen Anspruchsgruppen der Marke verschmelzen im digitalen Raum. (Potenzielle) Kundinnen und Kunden, Fans der Marke, Kritiker, Mitarbeitende des Unternehmens, Aktionäre oder Journalistinnen interagieren mit Marken (oft) über dieselben digitalen Kanäle und werden dabei auch mit denselben Inhalten konfrontiert (siehe etwa Valentini, 2015).

Es lässt sich festhalten, dass die verschiedenen Teilbereiche der Unternehmenskommunikation zunehmend zusammenfliessen und nur in ihrer Einheit eine erfolgreiche Markenkommunikation ermöglichen. Das zeigt sich auch organisatorisch. Unternehmen richten sogenannte Newsrooms ein, in denen die gesamte Marken- oder Unternehmenskommunikation zentral geplant wird (Moss, 2016; Spachmann \& Huck-Sandhu, 2019). Im Rahmen dieser Arbeit sollen deshalb Markenbotschaften als Botschaften der Unternehmenskommunikation verstanden werden. Damit wird den Überlegungen von Huck-Sandhu und Kirchenbauer (2017) gefolgt, die Markenbotschaften als «Corporate Messages» (S. 362) konzipieren. Corporate Messages sind dabei «Unternehmensbotschaften, die aus der Unternehmens- bzw. Kommunikationsstrategie abgeleitet und aufeinander abgestimmt formuliert sind» (Huck-Sandhu, 2014, S. 652). ${ }^{8}$

\footnotetext{
${ }^{8}$ Anzumerken ist, dass die Markenkommunikation nur einen Teilbereich der Unternehmenskommunikation ausmacht. Die Unternehmenskommunikation trägt durch ihren breiten Geltungsanspruch in verschiedener Weise zum Erreichen der strategischen Unternehmensziele bei (Zerfass, 2010, S. 290).
} 


\subsubsection{Inhaltliche Umorientierung}

Neben der eben präsentierten gedanklichen Umorientierung muss sich die Markenkommunikation auch inhaltlich umorientieren. Bolliger und Koob (2015) sprechen von einem Content Turn und meinen damit eine Neuorientierung in der Markenkommunikation, mit welcher «informierende, beratende oder auch unterhaltende und journalistisch geprägte Inhalte für die Zielgruppen» (S. 459) und Anspruchsgruppen in den Fokus rücken. Ein solch journalistisch geprägter, auf Geschichten und Inhalten beruhender Ansatz der Markenkommunikation kann als Antithese zur klassischen Markenkommunikation gesehen werden (Bolliger \& Koob, 2015). Als einen wesentlichen Treiber der Neuorientierung hin zum «inhaltsgetriebenen Ansatz» machen die Autoren die zunehmende Skepsis der Konsumentinnen und Konsumenten gegenüber (digitaler) Werbung aus. Empirische Resultate der Autoren (Bolliger \& Koob, 2015) zeigen zudem, dass sich der inhaltsgetriebene Ansatz in den Augen von über 300 befragten Kommunikationsentscheidern besser zur Erreichung von Kommunikationszielen eignet. $60 \%$ der befragten Fachpersonen erachten den inhaltsgetriebenen Ansatz als besser geeignet, um das Markenimage zu verbessern. Nur $6 \%$ sehen bei dieser Aufgabe den klassischen, werbeorientierten Ansatz im Vorteil. Die Produktion relevanter Inhalte im Sinne dieses «inhaltsgetriebenen» Ansatzes stellt für die Unternehmen jedoch eine grosse Herausforderung dar. Nur $19 \%$ der befragten Unternehmen verfügen über eine klar definierte Content-Strategie (Bolliger \& Koob, 2015).

Das Sammelbuch von Baetzgen und Tropp (2013) mit dem Titel «Brand Content - die Marke als Medienereignis» bringt diese Realität treffend zum Ausdruck. Im Vorwort zu ihrem Sammelband beschreiben die beiden diese neue Realität. Für die konstante, rund um die Uhr stattfindende Interaktion mit den Zielgruppen in einer digitalen Welt benötigen Marken relevante Inhalte. Vorbei seien die Zeiten, «als Marken nur in TV-Spots, Anzeigen oder Postern präsent waren» (Baetzgen \& Tropp, 2013, S. V). Für von Matt (2008) steht die Markenkommunikation zunehmend «im Wettbewerb mit Programminhalten» und muss bezüglich «Unterhaltung, Humor und Charme» (S. 9) mit diesen mithalten. Markenkommunikation und Werbung dürfen nicht länger nur aus Unternehmenssicht, sondern müssen zwingend auch aus Usersicht betrachtet werden.

An dieser Stelle muss eingewendet werden, dass es solche Überlegungen schon früher gab. Ideen und Umsetzungen im Sinne von «Brand Content» finden sich seit über 100 Jahren. Bekannte Beispiele früher Formen von Brand Content sind die von Procter und Gamble verfassten Comic-Geschichten in Sonntagszeitungen zur Bewerbung von Seife oder der von Michelin herausgegebene «Guide Michelin» mit «gastronomischen Ausflugstipps» (Burkhardt \& Siefke, 
2013, S. 135). Burkhardt und Siefke sehen auch in den Fugger-Zeitungen des 15. Jahrhunderts eine Form von Brand Content. Der Kaufmann Jakob Fugger informierte mit den Zeitungen seine Kundschaft über das Wirtschaftsgeschehen, integrierte aber auch unterhaltende Geschichten. Daneben gab es immer schon Bemühungen, Werbungen unterhaltsam zu gestalten. Die Kombination der englischen Begriffe «Advertisement» und «Entertainment» führte zum Konzept «Advertainment». Die Anfänge von Advertainment, so Hallahan (2018, S. 4), reichen bis in die 1920er-Jahre zurück, als Unternehmen begannen, Radio- oder Comedy Shows zu produzieren. Laut Knop (2006, S. 110) wurden Konzepte wie Advotainment oder Advertainment insbesondere ab Anfang der 1990er-Jahre benützt, um dem zunehmenden Unterhaltungswert von Werbung Ausdruck zu verleihen. Auch Schmidt (2004, S. 97) spricht von einer verstärkten Tendenz zur unterhaltenden Werbung ab den 1980er-Jahren.

Aus mindestens zwei Gründen ist es dennoch sinnvoll und passend, von einem Content Turn auszugehen:

1. Der Mehrwert für User findet gleichberechtigt Eingang in die Botschaft. Die Marken- oder Werbebotschaft steht nicht mehr allein im Zentrum der Kommunikation. Eine unterhaltsam gestaltete Werbung wird dieser Anforderung nicht zwingend gerecht.

2. In der digitalen Welt wird diese Form der Kommunikation zum Standard.

Im Folgenden wird diese Art der digitalen Markenkommunikation näher betrachtet. Zuerst wird aus einer marketingorientierten Perspektive auf die Begriffe Content Marketing und Brand Content eingegangen. Danach folgt aus einer kommunikationswissenschaftlichen Perspektive eine Betrachtung der Konzepte Content-Strategie und Themenmanagement.

\subsubsection{Content Marketing und Brand Content}

Das wohl bekannteste Konzept, das dem Content Turn entsprungen ist, ist Content Marketing (Hollebeek \& Macky, 2019). Pulizzi (2014) definiert Content Marketing als «the marketing and business process for creating and distributing valuable and compelling content to attract, acquire, and engage a clearly defined and understood target audience - with the objective of driving profitable customer action» (S. 5). In ähnlicher Weise ist nach Holliman und Rowley (2014) «Digital Content Marketing» eine Aktivität, bei welcher Inhalte «kreiert, kommuniziert, distribuiert und ausgetauscht werden» (S. 287), die sowohl für 
die Kunden als auch für die Marke einen Mehrwert haben. Beide Definitionen kombinieren eine marketingorientierte Unternehmensperspektive mit einer nutzenbringenden Userperspektive. Obwohl sich Content Marketing als zentraler Begriff etabliert hat, werden ähnliche Ideen auch unter anderen Schlagwörtern diskutiert. Tropp (2014a) etwa spricht von «Utility Marketing» (S. 1116) und meint damit Kommunikationsangebote mit Rezeptionsrelevanz. Nach Puligadda, DelVecchio und Gilbreath (2012) zeichnen sich Inhalte des «Meaningful Marketing» dadurch aus, dass sie (a) das Kundenerlebnis nicht unterbrechen oder stören, (b) nicht werberisch sind und (c) durch den Kunden sofort und ohne Gegenleistung konsumiert werden können. Hallahan (2018, S. 3) wiederum spricht primär von «Branded Content» und meint damit Inhalte, die selbstständig durch ein Unternehmen im Rahmen einer Content-Marketing-Initiative erstellt werden.

Unabhängig vom Grundkonzept sind zwei Fragen zentral:

- Wie wird die Userperspektive - also ein Mehrwert, ein Nutzen oder Relevanz für die Zielgruppe - ausgestaltet?

- Wie werden die Unternehmens- und die Userperspektive kombiniert?

Die erste Frage, also die Definition dieses Mehrwerts, stellt nach vor eine Herausforderung dar (Bolliger \& Koob, 2015, S. 464). Eine vor allem in der praxisorientierten Literatur beliebte Version liegt darin, den Mehrwert über Beispiele zu fassen (Hilker, 2017, S. 49). Eine ebenfalls oft genannte Taktik oder Strategie zur Herstellung relevanter Inhalte ist Storytelling (Tillmanns, 2018, S. 306). Bei Storytelling werden Kommunikationsinhalte in eine Geschichte integriert. Die Geschichten wiederum folgen einem oftmals gleichbleibenden Muster, wonach ein Protagonist erfolgreich eine Herausforderung, Hürde oder ein Problem bewältigt (Demarmels et al., 2018, S. 101). Für eine Festlegung des Mehrwertes sind beide Varianten unzureichend. Bei der Nennung erfolgreicher «Content»-Beispiele fehlt eine systematische Auseinandersetzung mit dem, was den «Mehrwert» tatsächlich ausmacht. Zwar werden auch systematische Inhaltsanalysen digitaler Markenbotschaften vorgenommen, diese beziehen in der Untersuchung aber sämtliche Botschaften mit ein und gehen nicht primär auf Botschaften im Sinne des Content Marketings ein. Storytelling beantwortet primär die Frage, wie ein Inhalt als Geschichte aufbereitet werden soll. Der Inhalt der Geschichten wird nicht prioritär behandelt.

Vielversprechender scheint die Annäherung an den «Mehrwert» über den Usesand-Gratifications-Ansatz (U\&G-Ansatz; H. Schramm \& Knoll, 2013; Tropp, 2014a). In dieser Tradition wird insbesondere auf «Information» und «Unterhaltung» als relevante Gratifikationen des Medienkonsums zurückgegriffen, um 
einen Mehrwert durch «Content» inhaltlich zu fassen (Hallahan, 2018, S. 3; Lou et al., 2019, S. 775; Wall \& Spinuzzi, 2018, S. 137). Wenn Menschen sich wegen Information und Unterhaltung freiwillig Medien zuwenden, dann, so die Annahme, werden sie sich auch Markeninhalten zuwenden, sofern diese ebenfalls Information und Unterhaltung bieten.

Just as advertising has been associated with the goals of informing and/or entertaining, so too is content marketing. The content marketing aspects of social publishing are focused on the goals of informing. The content marketing aspects of social entertainment are focused on the goal of entertaining. (Tuten \& Solomon, 2015, S. 203)

Tuten und Solomon (2015, S. 202) verstehen Content Marketing der obigen Definition nach als Oberbegriff für Branded Entertainment und Social Publishing. Branded Entertainment sind Inhalte, z. B. in Form eines Spiels oder Films, welche die Aufmerksamkeit der Zuschauer wecken und über längere Zeit aufrechterhalten, indem sie die Zuschauer unterhalten. Die Inhalte werden durch das Unternehmen produziert (Tuten \& Solomon, 2015, S. 202-203). Bei Social Publishing geht es darum, Informationen zu vermitteln und einen Standpunkt zu einem relevanten Thema zum Ausdruck zu bringen (S. 203). In Anlehnung an den Blogger Yaro Starak (2008) nennen die Autoren (S. 175) «How-To Artikel, Definitionen, Glossar-Artikel, theoretische Artikel und Listen/Aufzählungen» als Formen des Social Publishing. Die Inhalte können unterschiedlichste Formen und Formate annehmen und dabei auch lustig oder inspirierend sein (S. 173). Insofern unterscheiden sich Branded Entertainment und Social Publishing zwar in ihrer groben Ausrichtung hinsichtlich Unterhaltung und Information, es findet aber keine strikte Trennung der Konstrukte statt. Vor dem Hintergrund, dass sich Information und Unterhaltung nicht ausschliessen (Bosshart, 2007, S. 19), ist das plausibel.

Für Kunz und Elsässer (2016) zeichnet sich Branded Entertainment durch die Verschmelzung journalistischer und markenbezogener Inhalte aus. Durch seinen «unterhaltenden Charakter» (S. 53) eignet sich Branded Entertainment, um die Kunden trotz der Vielzahl an digital vermittelten Kommunikationsbotschaften zu erreichen (Kunz \& Elsässer, 2016). Gerade der Begriff «Branded Entertainment» - im Sinne von «markierter» Unterhaltung - wird oftmals auch verwendet, um unterhaltende Inhalte Dritter zu bezeichnen, in welche Produkte, Marken oder Werbebotschaften integriert werden (Hudson \& Hudson, 2006; Pelsmacker, 2016, S. xiv). Product Placement kann demnach als eine Art von Branded Entertainment verstanden werden. 
Als Zwischenfazit zur ersten Frage kann festgehalten werden, dass die Annäherung an den Mehrwert am besten über den Uses-and-Gratifications-Ansatz gelingt. Der Mehrwert wird demnach insbesondere über «Information» und «Unterhaltung» erreicht.

Die zweite wesentliche Frage in Bezug auf Content Marketing und Brand Content dreht sich darum, in welchem Verhältnis werberische Aussagen und der Mehrwert für den User stehen. Dass darüber keine Einigkeit herrscht, zeigen beispielsweise diverse Aussagen aus dem Sammelband «Brand Content» von Baetzgen und Tropp (2013). Die beiden Herausgeber schreiben (Tropp \& Baetzgen, 2013): «Der Unterhaltungs- und Informationswert steht deshalb an erster Stelle - die Markenbotschaft steht dagegen hinten an» (S. 11). Eine ähnliche Sichtweise vertreten Albers und Hanke (2013) und raten davon ab, die Marke in den Vordergrund zu stellen: «Erst wenn Marken aufhören, stets zuerst Marken sein zu wollen, werden sie kommunikativ erfolgreich sein» (S. 33). An anderer Stelle sind Baetzgen und Tropp (2013) dann etwas optimistischer in Hinsicht auf Markenbotschaften: «Deshalb ist es wichtig, dass eine Marke Themen definiert, die sie glaubwürdig besetzen kann - als Marke und als Medium. Meist ergeben sich diese Themen aus dem Produkt, der Produkteverwendung oder den Interessen der Verwender» (S. 11-12). Die Themen werden hier also nicht nur von der Marke her abgeleitet, sondern finden sich im Idealfall rund um ihre Produkte und Dienstleistungen. Figge und Albers (2013) pflichten bei und meinen, «die für gelungenes Content Marketing entscheidenden Inhalte beginnen bei dem, was eigentlich schon vorhanden ist [...]: Informationen, die Interessenten helfen, sich ein klares und konsistentes Bild über das Unternehmen, seine Produkte und Leistungen zu machen» (S. 92). Für Kreutzer und Land (2017) sollen «informierende, beratende und/oder unterhaltende Inhalte» (S. 157) des Content Marketings nur indirekt auf die Produkte und Dienstleistungen des Unternehmens verweisen und keine direkten Kaufimpulse setzen. Dennoch sollen die Kunden über Content Marketing an das Unternehmen herangeführt werden, was mittelfristig zu Verkäufen führt. Zudem sollen die Inhalte auch «die unternehmerische Kompetenz» (S. 158) aufzeigen. Die Ausführungen zeugen von der verwirrenden Lage, geben allerdings wenig operative Hilfestellung dahingehend, wie eine Unternehmensperspektive und eine Userperspektive in einer Brand-Content-Botschaft systematisch kombiniert werden können.

Zur zweiten Frage lässt sich deshalb festhalten, dass Brand Content im Idealfall eine Verbindung zur Marke, den Produkten oder Dienstleistungen haben soll. Wie nah diese Verbindung sein soll und wie diese auszugestalten ist, darüber herrscht in der Literatur aber keine Einigkeit. 


\subsubsection{Content-Strategie und Themenmanagement}

Aus der Perspektive der Public Relations oder der Unternehmenskommunikation wird vermehrt von Content-Strategie oder Themenmanagement gesprochen als von Content-Marketing (Huck-Sandhu \& Kirchenbauer, 2017). Allerdings tut sich auch dieser Literaturstrang mit der Operationalisierung des «Mehrwertes» nicht nur leicht. Im Buch «Social Media Campaigns» von C. M. Kim (2016) finden sich etwa nur wenig Orientierungspunkte zur inhaltlichen Ausgestaltung von Botschaften. Unter «Creative Strategies» geht die Autorin auf verschiedene Taktiken ein, mit denen eine Marke in Communitys positioniert werden kann (C. M. Kim, 2016, S. 106-117). Diese Taktiken sind abstrakt gehalten und raten dazu, glaubwürdig zu kommunizieren, Beziehungen oder Engagement aufzubauen. Die Taktik «Brand Expertise» kann am ehesten im Sinne einer inhaltlichen Hilfestellung dienen. Dieser Taktik gemäss sollen sich Marken als Experten und Vordenker positionieren und relevante Neuigkeiten und Informationen aus ihrem Tätigkeitsbereich an die Userinnen und User weitergeben. Dazu würden sich insbesondere Infografiken oder Publikationen eignen (C. M. Kim, 2016, S. 108-109).

Arrese und Pérez-Latre (2017) legen den Fokus ihrer Betrachtungen auf Brand Journalismus und verstehen diesen als Teilbereich des Content Marketings. Nach den Autoren geht es bei Brand Journalismus darum, «Brand Content» (S. 126) aus «journalistischer Perspektive» (S. 122) aufzubereiten und über eigene Kanäle zu vertreiben. Die Inhalte sollen einen Mehrwert und Neuigkeitswert bieten. Ziel solcher Inhalte ist es, Beziehungen zu Kunden und Stakeholdern aufzubauen, aber auch Autorität und Einfluss im Markt und in der Gesellschaft zu gewinnen (S. 124). Anstatt über die eigenen Produkte, deren Nutzen oder Unternehmenswerte zu sprechen, müssen Marken einen themenbezogenen Ansatz einnehmen und «Diskussion, Partizipation und Engagement» fördern (S. 127). Der Vermerk auf die «journalistische Perspektive» kann als Orientierung beim Erstellen von Inhalten dienen - die Autoren führen unter anderem auch aus, was «journalistisch» impliziert (S. 129) -, bietet aber keine inhaltliche Hilfestellung. Am ehesten kann der Begriff «Neuigkeitswert» (S. 124) einen Hinweis auf die Ausgestaltung eines Mehrwerts für die User liefern.

Für Mast (2019) geht es bei Content Management um die «Aufbereitung attraktiver Themen» (S. 233). Inhalte oder Themen sollen für die Menschen nützlich sein, ihnen beispielsweise im Alltag Orientierung bieten (S. 234) und «Zugang zur Lebenswelt der Menschen» (S. 244) finden. Dazu können Unternehmen verschiedene redaktionelle Strategien einsetzen (Mast, 2019, S. 262). Bei der «Strategie der Ereignisorientierung» geht es um die Vermittlung von Fakten und 
Wissen mit Neuigkeitswert. Bei der «wissenszentrierten Strategie» stehen Zusammenhänge und Hintergrundwissen im Fokus. Die «handlungszentrierte Strategie» bietet Ratschläge bei Problemen und Entscheidungssituationen. Die «gefühlszentrierte Strategie» vermittelt anhand von Geschichten Emotionen. Bei der durch Unternehmen spärlich eingesetzten «skandalorientierten Strategie» wird versucht, «für Aufsehen und Aufregen zu sorgen» (S. 262). Das Content Management als Teil der Unternehmenskommunikation sorgt damit für den Aufbau und die Pflege langfristiger Beziehungen des Unternehmens mit den Anspruchsgruppen (Mast, 2019, S. 235). Mast zieht damit auch eine Trennlinie zu Content Marketing, das sich ihrer Sicht nach insbesondere an Kunden wendet und auf kurzfristige Effekte aus ist (S. 235).

Sehr präzise setzen sich auch Huck-Sandhu und Kirchenbauer (2017) mit dem Thema Content-Strategie auseinander. Die Content-Strategie identifiziert «Leit- und Kernthemen im Kontext der Markenkommunikation» (S. 365). Themen müssen einerseits im Einklang mit der Unternehmens- bzw. Markenstrategie stehen und andererseits die Interessen der Stakeholder bedienen (Huck-Sandhu \& Kirchenbauer, 2017, S. 365). Aus der Content-Strategie können Corporate Messages (Markenbotschaften) abgeleitet werden. Diese wiederum können aus einer Inhaltsperspektive (was bzw. welcher Inhalt wird kommuniziert?) und einer Aufbereitungsperspektive (wie werden die Inhalte kommuniziert?) betrachtet werden. Huck-Sandhu und Kirchenbauer unterscheiden - mit Bezug auf die Journalistik - fünf verschiedene Kommunikationsmodi: informierender, erklärender/argumentierender, bewertender, narrativer und diskursiver Modus. Die ersten drei Modi behandeln insbesondere die Frage, welche Inhalte kommuniziert werden, und können für eine einfachere Gliederung unter dem «informierenden Modus» zusammengefasst werden (Huck-Sandhu \& Kirchenbauer, 2017, S. 368). Die Vermittlung von Produktinformationen, von relevantem (Hintergrund-)Wissen, von Fakten oder von Erklärungen steht im Zentrum dieses ersten (zusammengefassten) Modus. Beim narrativen Modus handelt es sich hingegen um «eine spezifische Form der Vermittlung», die insbesondere auf Erzählungen, Geschichten und Emotionen zurückgreift. Dialoge machen schliesslich den diskursiven Modus aus.

In einem weiteren Schritt bringen auch Huck-Sandhu und Kirchenbauer die Kommunikationsmodi mit den Nutzungsmotiven im Sinne des U\&G-Ansatzes überein. Der informierende Modus, so die Autorinnen, eigne sich insbesondere, um Informationsbedürfnisse von Kunden, z. B. in Bezug auf Produkte oder Services, zu befriedigen. Der narrative Modus eignet sich sowohl für Informationsals auch für Unterhaltungsbedürfnisse. Er «verbindet die Informationsvermittlung mit der Unterhaltung der User» (Huck-Sandhu \& Kirchenbauer, 2017, S. 372). Der diskursive Modus eignet sich für Diskussions- und Austauschbedürfnisse der User. 
Die Ausführungen von Mast (2019) und von Huck-Sandhu und Kirchenbauer (2017) sind in vielerlei Hinsicht wertvoll. Den Ausführungen von Huck-Sandhu und Kirchenbauer kann entnommen werden, dass Information sowohl aus Produktinformation als auch aus weiterführender Hintergrundinformation bestehen kann. Auch Mast sieht in der Vermittlung von Hintergrundwissen, Ratschlägen und Fakten einen Mehrwert. Damit ergeben sich aus diesen Ausführungen erste Hinweise darauf, wie ein Mehrwert im Sinne der «Information» ausgestaltet werden könnte. Unterhaltung (Huck-Sandhu \& Kirchenbauer, 2017) oder Emotionalität (Mast, 2019) kann hingegen durch eine narrative Erzählweise entstehen. Während Huck-Sandhu und Kirchenbauer Informationen als Grundlage dieser Erzählweise sehen, geht Mast nicht weiter auf die inhaltlichen Aspekte ein. Das inhaltliche Wesen von Unterhaltung als Mehrwert bleibt damit vorerst ungeklärt. Auch stellt sich nach wie vor die Frage nach der Kombination der Unternehmens- und der Userperspektive. Können Hintergrundinformationen zum Produkt als Brand Content gewertet werden? Ist das Erzählen eines Witzes Brand Content, weil damit potenziell Unterhaltung geboten wird?

\subsection{Brand Content: Forschungsbedarf und Ausblick}

Im Rahmen dieser Arbeit soll der Einfluss von Brand Content auf das Markenimage analysiert werden. Es wird der Term «Brand Content» und nicht «Branded Content» verwendet, um auszudrücken, dass es sich um unternehmensgenerierte Inhalte handelt, die in eigenen Kanälen (owned media) publiziert werden. «Branded Content» hingegen stünde für gesponserte und bezahlte Inhalte, die in die (redaktionellen) Inhalte Dritter eingebunden werden (Arrese \& Pérez-Latre, 2017, S. 134). Auf den Begriff «Content Marketing» wird verzichtet, weil er primär einer Marketing-Denkweise folgt und sich in erster Linie an Kunden richtet (Mast, 2019, S. 235). In Abschnitt 2.4.1 wurde entgegen dieser Denkweise dargelegt, dass Markenbotschaften als Botschaften der Unternehmenskommunikation verstanden werden sollen. Zusammenfassend lässt sich Brand Content folgendermassen charakterisieren:

Als Brand Content sind im Rahmen dieser Arbeit Botschaften der Unternehmenskommunikation zu verstehen, die gleichberechtigt eine Unternehmensperspektive (Imageaufbau) als auch eine Userperspektive (Mehrwert für User) umfassen und primär über digitale Kanäle vermittelt werden. 
Die Kommunikation aus der Unternehmensperspektive ist gut erforscht und ausgearbeitet (siehe auch Abschnitt 2.3.5). Es können eine informative und eine emotionale Kommunikationsstrategie unterschieden werden, die den Aufbau eines funktionalen bzw. emotionalen Markenimages zum Ziel haben. Die Fragen, inwiefern der Mehrwert von Brand Content - also die Userperspektive - festgemacht wird und wie die Unternehmensperspektive und die Userperspektive sinnvoll zusammenfliessen, sind jedoch noch nicht restlos geklärt. Deshalb wird in einem nächsten Kapitel der Uses-and-Gratifications-Ansatz, näher beleuchtet. Diese Detailbetrachtung soll helfen, den inhaltlichen Mehrwert von Brand Content besser zu fassen und - sollten sich die beiden Dimensionen in ihrer Relevanz bestätigen - Information und Unterhaltung zu definieren. Sobald der Mehrwert (Userperspektive) inhaltlich geklärt ist, kann eine sinnvolle Kombination mit der Unternehmensperspektive erfolgen. Dieses Vorgehen erlaubt es, Brand Content zu fassen und dessen Auswirkungen auf das Markenimage zu untersuchen.

Die vorliegende Studie - das wurde in der Einleitung im Detail dargelegt ist relevant, weil Brand Content im Allgemeinen und dessen Auswirkungen auf das Markenimage im Speziellen ungenügend erforscht sind (Hollebeek \& Macky, 2019; Lou et al., 2019; Müller \& Christandl, 2019; Wall \& Spinuzzi, 2018).

Open Access Dieses Kapitel wird unter der Creative Commons Namensnennung 4.0 International Lizenz (http://creativecommons.org/licenses/by/4.0/deed.de) veröffentlicht, welche die Nutzung, Vervielfältigung, Bearbeitung, Verbreitung und Wiedergabe in jeglichem Medium und Format erlaubt, sofern Sie den/die ursprünglichen Autor(en) und die Quelle ordnungsgemäß nennen, einen Link zur Creative Commons Lizenz beifügen und angeben, ob Änderungen vorgenommen wurden.

Die in diesem Kapitel enthaltenen Bilder und sonstiges Drittmaterial unterliegen ebenfalls der genannten Creative Commons Lizenz, sofern sich aus der Abbildungslegende nichts anderes ergibt. Sofern das betreffende Material nicht unter der genannten Creative Commons Lizenz steht und die betreffende Handlung nicht nach gesetzlichen Vorschriften erlaubt ist, ist für die oben aufgeführten Weiterverwendungen des Materials die Einwilligung des jeweiligen Rechteinhabers einzuholen.

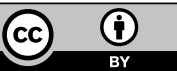

